\title{
Miscommunication Detection and Recovery in Situated Human-Robot Dialogue
}

\author{
MATTHEW MARGE, U.S. Army Research Laboratory \\ ALEXANDER I. RUDNICKY, Carnegie Mellon University
}

\begin{abstract}
Even without speech recognition errors, robots may face difficulties interpreting natural-language instructions. We present a method for robustly handling miscommunication between people and robots in task-oriented spoken dialogue. This capability is implemented in TeamTalk, a conversational interface to robots that supports detection and recovery from the situated grounding problems of referential ambiguity and impossible actions. We introduce a representation that detects these problems and a nearest-neighbor learning algorithm that selects recovery strategies for a virtual robot. When the robot encounters a grounding problem, it looks back on its interaction history to consider how it resolved similar situations. The learning method is trained initially on crowdsourced data but is then supplemented by interactions from a longitudinal user study in which six participants performed navigation tasks with the robot. We compare results collected using a general model to user-specific models and find that user-specific models perform best on measures of dialogue efficiency, while the general model yields the highest agreement with human judges. Our overall contribution is a novel approach to detecting and recovering from miscommunication in dialogue by including situated context, namely, information from a robot's path planner and surroundings.
\end{abstract}

CCS Concepts: - Computing methodologies $\rightarrow$ Discourse, dialogue and pragmatics; • Humancentered computing $\rightarrow$ Natural language interfaces;

Additional Key Words and Phrases: Human-robot communication, human-robot interaction, physically situated dialogue, spoken-dialogue systems, language grounding

\section{ACM Reference format:}

Matthew Marge and Alexander I. Rudnicky. 2019. Miscommunication Detection and Recovery in Situated Human-Robot Dialogue. ACM Trans. Interact. Intell. Syst. 9, 1, Article 3 (February 2019), 40 pages.

https://doi.org/10.1145/3237189

\section{INTRODUCTION}

In recent years, advances in robotics, computer vision, and autonomous navigation have brought robots out of labs and into real-world spaces. The emergence of mobile, physically situated agents like robots has also created a desire to communicate with them effectively. Dialogue offers a promising way to interact with robots: a hands-free mode of communication that conveys information at a more abstract task level than typical command-and-control devices [34]. With

The reviewing of this article was managed by associate editor Joyce Chai.

This work was sponsored by the Boeing Company, a National Science Foundation Graduate Research Fellowship, and the U.S. Army Research Laboratory.

Most of this work was conducted while the first author was a student at Carnegie Mellon University.

Authors' addresses: M. Marge, Computational and Information Sciences Directorate, U.S. Army Research Laboratory, 2800 Powder Mill Road, Adelphi, MD 20783; email: matthew.r.marge.civ@mail.mil; A. I. Rudnicky, School of Computer Science, 5000 Forbes Avenue, Pittsburgh, PA 15213; email: air@cs.cmu.edu.

Publication rights licensed to ACM. ACM acknowledges that this contribution was authored or co-authored by an employee, contractor or affiliate of the United States government. As such, the Government retains a nonexclusive, royaltyfree right to publish or reproduce this article, or to allow others to do so, for Government purposes only.

(C) 2019 Copyright held by the owner/author(s). Publication rights licensed to ACM.

2160-6455/2019/02-ART3 \$15.00

https://doi.org/10.1145/3237189 
communication being bidirectional, interlocutors can provide status updates to each other and prompt for clarification so that misunderstandings can be resolved [105]. Physically situated dialogue is dialogue that relies on context from the physical world: from a robot's perspective, a streaming source of sensor data that is often crucial for accomplishing tasks [11]. In this article, we present a research framework for handling miscommunication between people and robots in situated dialogue. Our overall contribution is a novel approach to incorporating information from situated context-that is, a robot's path planner and its surroundings-to detect and recover from miscommunication. We conducted a longitudinal evaluation with six participants and a virtual robot using a nearest-neighbor algorithm for learning recovery strategies. We found improvements in dialogue efficiency as the robot accumulated experience with the task.

The focus of this work is on robot navigation, a domain that can involve communication about distance, space, and referable objects in the environment. To address challenges in this domain, researchers have studied situated interaction [54]: communication between two or more communicative partners that uses environment context to accomplish tasks. Situated reasoning is comprised of the core capabilities necessary to operate effectively in these interactions. We introduce an approach to dialogue systems that leverages situated reasoning so that robots can be more resilient to miscommunication when doing navigation tasks.

The nature of situated interaction yields a relatively unexplored set of problems separate from traditional human-computer dialogue. Task requests may fail owing to dependencies on specific environment configurations, such as when a robot's path to a goal is blocked. These environments are often changing, and people may make unrealistic assumptions about a robot's ability to monitor its surroundings. Without help from people, a robot would have to act exclusively on noisy representations that could lead to further system failures.

Central to human-robot miscommunication are situated grounding ${ }^{1}$ problems [73], which happen when a robot fails to uniquely map a person's instruction to its surroundings, even though the spoken language may have been correctly recognized. Consider the case in which a human operator tells a robot to search a nearby room (e.g., "Search the room on your left"). If the operator does not provide enough detail about the room, such as when there is more than one room on the robot's left, there is a referential ambiguity problem; the robot could produce multiple navigation plans for the instruction. In contrast, if the robot detects no rooms on its left or all of the rooms are inaccessible, there is an impossible-to-execute problem; the robot cannot produce a single plan that uniquely maps the user's instruction to its surroundings. Finally, instructions that resolve to a single plan are executable.

This article describes an infrastructure and representation that permits a robot to use spoken dialogue to robustly detect and recover from miscommunication. First, in Section 2, we discuss the requirements for detecting situated grounding problems in robot navigation. In Section 3, we review related work. In Section 4, we describe TeamTalk, a conversational interface to robots that was used in this work. In Section 5, we describe our approach to detecting situated grounding problems with the Situated Reasoner, an extension to TeamTalk. In Section 6, we then present recovery strategies that enable robots to recover from these types of problems based on an analysis of human-human dialogue and the problem space. Section 7 contains a discussion on improving recovery strategy decision-making. In Section 8, we present our findings from real-time experimentation with recovery learning. Finally, in Section 9, we present an evaluation of a nearestneighbor approach to learning which recovery strategies a robot should use in different situations.

\footnotetext{
${ }^{1}$ Grounding here refers to grounding in communication [28], the process by which two dialogue partners accumulate information in their common ground. It does not refer to symbol grounding, which has become a popular term in the robotics literature for aligning words to sensory perception [42]
} 
In a study with six participants, we find improvements in performance over time on measures of human-robot dialogue efficiency, with user-specific models outperforming a general model that leveraged all dialogue interactions. However, results indicate that the general model's recovery strategy selection agrees more with human judges than user-specific models, showing that gathering a variety of user preferences can also help.

We make the following contributions:

- A representation for detecting situated grounding problems that analyzes features from spoken language input, the robot's path planner, and the robot's surroundings (Section 5).

- Definition of recovery strategies for physically situated dialogue (Section 6).

- A learning algorithm that (1) changes how a robot selects recovery strategies for situated grounding problems over time (Section 7), and (2) is evaluated in a longitudinal user study with six participants (Section 8) and by computing agreement with human judges (Section 9).

\section{BACKGROUND}

In this section, we introduce the task domain in which we investigate miscommunication in physically situated dialogue and describe the theoretical foundations and capabilities needed to perform grounding at multiple levels of understanding.

\subsection{Directed Navigation Task}

There are many tasks in which robots can interact with the physical world [11]. In this work, we define a directed navigation task in which a human issues route instructions and a robot follows them. This was meant to focus on where situated grounding problems commonly occur. Mobile robots must master navigation if they are to work with people-successful interactions often require a robot to move around an environment based on a user's instructions. The nature of route instructions also make them a good candidate for automatic interpretation. Route instructions are sequential, meaning that the order in which instructions are presented is how they should most often be executed [57]. Evaluation with route instructions works well in collaborative tasks because the user's intention is largely matched if the robot arrives at a specified destination (correct subgoals may or may not be important). Other possible measures can optimize for distance from the goal, paths toward the goal, and time elapsed. Route instructions also follow a predictable structure and can be classified into distinct categories, such as imperative commands and side comments [87]. In essence, route instructions form a sublanguage of their original language, which precludes out-of-domain errors.

We investigate a task domain that involves two dialogue partners, a human (the directiongiver), and a robot (a direction-follower). The direction-giver-follower paradigm in dialogue has seen a wide adoption, originating with the Human Communication Research Centre (HCRC) Map Task corpus [1] and continuing with other human-human and human-computer dialogue corpora (e.g., $[71,80,110])$. The direction-giver assigns the follower navigation tasks by communicating about space, referents, and distance as route instructions. Those tasks become the goals that the direction-follower must accomplish to achieve mutual task success. The direction-follower performs the giver's requested tasks, asking for clarification or help as needed.

\subsection{Levels of Understanding in Human-Robot Dialogue}

One aim of this work is to detect occurrences of miscommunication in situated human-robot dialogue. Detecting these requires monitoring for possible problems at various points of Clark's levels of understanding [27]. Figure 1 presents Clark's levels as adapted for human-robot 
LEVELS OF UNDERSTANDING

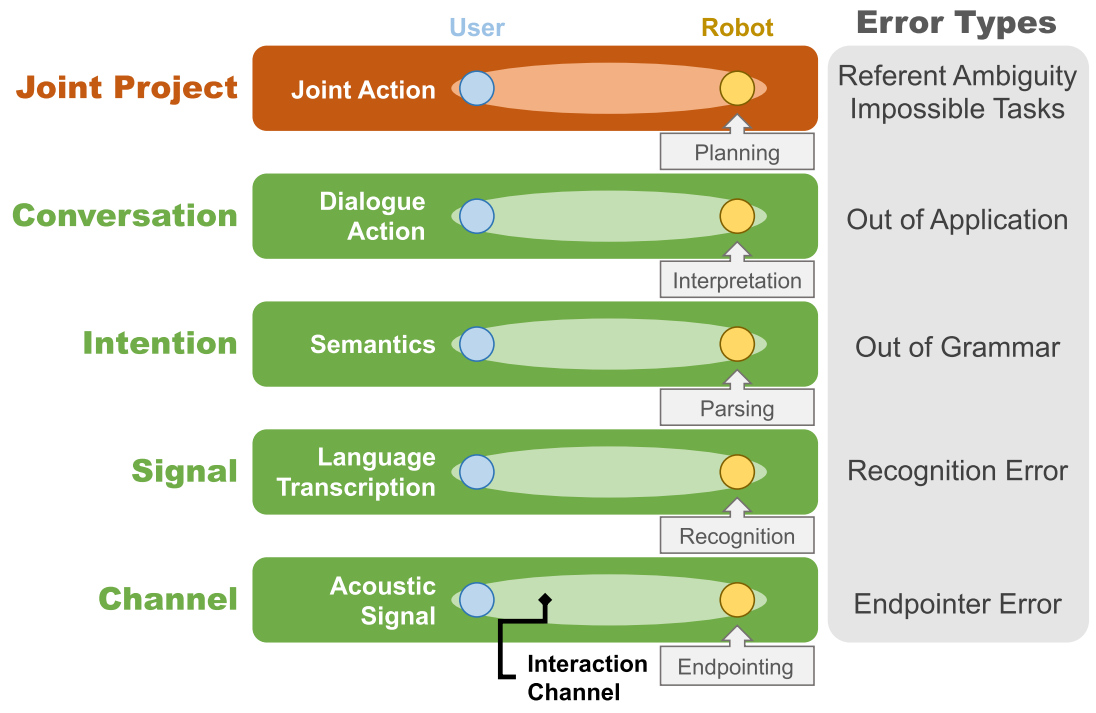

Fig. 1. Levels of understanding in human-robot dialogue, extended from $[10,27,85]$ to include the JoINT PROJECT level of understanding for handling errors such as referential ambiguities and impossible tasks.

communication, extending previous taxonomies by Paek [85] and Bohus [10]. Situated grounding problems happen at the highest level (the JOINT PROJECT level) because there may be too many possible plans (i.e., referential ambiguity in the context of performing an action in the environment) or none at all (i.e., impossible-to-execute instructions). Detecting these types of problems in situated dialogue has been limited to object manipulation to date [30].

Lower levels of understanding (CHANNEL, SIGNAL, INTENTION, and CONVERSATION) have received considerable attention in dialogue systems research. At the lowest point, the CHANNEL level consists of the user communicating speech as an acoustic signal to the robot. The robot must correctly endpoint the user's utterance; failure to do so, like cutting off a user mid-sentence, results in an endpointer error. As one example, Raux and Eskenazi [92] developed a method to optimize endpointing using dialogue features. Next, the SIGNAL level consists of the user communicating words to the robot. The robot's speech recognizer is responsible for transcribing the speech from an acoustic signal into words; the misinterpretation of words results in recognition errors. The INTENTION level consists of the user communicating intentions (i.e., semantics at the level of requested actions and other responses that drive the dialogue) to the robot. If the robot attempts to extract this information from speech recognizer output, any words that fail to parse are out of grammar errors. Traditionally spoken dialogue systems use context-free grammars or semantic parsers to define the semantics of any expected interactions with users. In related work, Bohus [10] and Skantze [106] used features from the SIGNAL and INTENTION levels to detect miscommunication in spoken-dialogue systems. The CONVERSATION level consists of the user communicating dialogue actions to the robot. Dialogue actions include commands, confirmations, and requests for information. Any dialogue action that falls beyond the scope of the robot's capabilities constitutes an out of application error.

Most dialogue systems can handle errors up to the conversation level of understanding. The JOINT PROJECT level sits above the other levels; it describes the overall joint action taking place in the dialogue at that moment, whether the user or robot is requesting the action. According 
to Clark [27], at this level the joint action is considered a joint project because one speaker is conveying an action that both dialogue partners undertake; they must coordinate their actions in order to complete the project. In human-robot dialogue, joint projects require the robot to plan out a series of actions to accomplish what the user requests. In turn, the robot should expect the user to monitor the situation, ensuring that the robot is doing the correct actions.

\subsection{Core Capabilities for Detecting Miscommunication in Situated Dialogue}

Any exchangeable information during the course of a dialogue can be used for grounding and, by extension, to detect miscommunication. That information can ground who has the conversational floor, along with the words, actions, and plans under discussion. A robot must maintain and update its notion of physically situated context over the course of interactions with a user. This requires monitoring the surroundings, user input, and any uncertainties associated with them. Dialogue partners always express some form of context as part of the grounding process, and Clark's levels of understanding [27] align with different sources of context. For example, clarifying words from speech-recognition output happens at the SIGNAL level, while clarifying ambiguous referents or impossible actions happens at the JOINT PROJECT level.

A robot must have several core capabilities to detect situated grounding problems at the JOINT PROJECT level. These capabilities include (1) task-independent and (2) physically situated skills that the robot uses to detect and repair problems at the JOINT PROJECT level. Managing and maintaining context requires task-independent skills (e.g., awareness of too few or too many choices to resolve a referent, knowing when to appeal to the user for help, awareness of poor speech-recognition confidence) and those skills specific to situated interaction (e.g., spatial reasoning, color detection). Task-independent skills can be activated from within a robot's dialogue manager and physically situated skills from within a reasoning component.

Task-Independent Skills. If a plan request originating from spoken language input results in more than one possibility, the robot assumes there to be a referential ambiguity. Resolving the referential ambiguity involves presenting one or more possible choices to the user. Detecting multiple possibilities and presenting the list of options to the user is a task-independent skill. The same can be said for detecting no possibilities from a user's request.

Physically Situated Skills. Task-dependent skills can vary; in this work, we consider only those for the directed navigation task. Physically situated skills in our work include awareness of proximity of some object to other objects or to the robot itself ("the door near you"); awareness of spatial properties of a referent relative to the robot ("the box on your left"); awareness of the location, color, or size of objects; and temporal information if it influences task execution.

\section{RELATED WORK}

Much of this research builds on related work on robots that ask for help, symbol grounding for natural language interpretation, dialogue systems that support human-robot interaction (HRI), and error handling in human-computer dialogue.

\subsection{Asking for Help}

Several recent papers have demonstrated the feasibility of symbiotic HRI, whereby a robot asks a human bystander or teammate for help, and the human helps due to some mutual benefit. The CoBot project [94] found that a robotic tour guide could significantly reduce its localization uncertainty by asking visitors for help. Deits et al. [30] developed an information theoretic method of asking for help using clarification dialogues to disambiguate referents. Their robot asked for 
clarifications about symbol grounding mappings between natural-language phrases and physical objects based on an estimate of uncertainty. Both of these approaches focused on a particular function that a robot could use to ask for help (e.g., knowing where it was in a building or if the phrase "pallet near the truck" referred to a specific location). In contrast, Knepper et al. [48] used inverse semantics to enable a robot to ask for targeted help on a construction task in which a human partner could provide assistance. The robot would autonomously detect when it entered a failure condition and took the human's perspective to determine how they could help and how to refer to the surroundings in natural language.

Although algorithms exist for requesting help, the robots that ask them either issue statements (no question generation) or interpret text input (no speech). Often, the robot can interpret only non-linguistic changes in its state space or inputs on a screen, performed by human teammates in response to a request. When questions are generated, they are focused on confirming a robot's certainty about a grounded symbol and not higher-level descriptions of referring expressions. As of this writing, none of these approaches handles requests for help when a robot receives an impossible-to-execute command.

The TeamTalk platform used in this work offers a bidirectional method for interpreting commands by detecting problems with task execution, requesting help, and responding to amended instructions from the human. The interaction modality is spoken dialogue; thus, the human is able to interact in a "heads-up, hands-free" way [69]. When disambiguating referring expressions, TeamTalk uses natural-language generation templates to describe its surroundings. Additionally, TeamTalk can detect impossible-to-execute commands, propose alternative referents, and prompt the human for clarification. Similar to previous work, users of TeamTalk have direct incentive to help the robot, given that the goal is to accomplish search and navigation tasks together. The above studies assessed interactions in fixed settings; in this article, we evaluate TeamTalk's ability to improve selection of dialogue strategies over time in longitudinal interactions over six sessions for each of the six participants.

\subsection{Route Instruction Interpretation}

The directed navigation task used in this research involves a human giving navigational directions, commonly termed route instructions, to a robot. Route instructions have been thoroughly studied, both in human-human $[1,100,110,112]$ and human-robot interactions (e.g., [51, 70, 87, 89, 103]). Bugmann et al. [20] coined the term corpus-based robotics to characterize the nature of the traditional research paradigm for route instruction interpretation: human-authored language is collected in experimental settings (ideally with the humans informed that the instructions are intended for a robot), the data is annotated, and a statistical model is trained for automatic interpretation. Route instruction interpreters generally take natural language as input and output robot-executable procedures.

Several groups have developed route instruction interpreters, some for written language beginning with MARCO [66] and continuing with an extensive body of follow-up work for navigation and manipulation tasks (e.g., [9, 16, 31, 33, 50, 62, 88]). Vogel and Jurafsky notably developed a route instruction follower using examples from the HCRC Map Task corpus [117]. Often, the work is framed to address the symbol grounding problem, whereby natural-language phrases represent symbols that a robot must map to its sensory perception. Some symbol grounding-based approaches interpret written language $[65,81,102,104,111]$. More recent techniques for route instruction interpretation include semantic parsing [3, 26, 53, 75], machine translation [74], and Tactical Behavior Specification Grammar [45]. A few approaches exist for interpreting spoken language, including Dzifcak et al. [32], Meena et al. [79], Bastianelli et al. [7], and our previous 
work [95]. Most recently, Broad et al. [19] developed a method for handling self-correcting monologue in spoken-language instructions.

The existing body of work does not cover handling interpretation failures for navigation, specifically supported by back-and-forth dialogue. Without interacting with the user, the best that such systems can do is estimate intention. In this article, we define methods that robots can use to detect these kinds of problems. We also present a set of dialogue strategies for robots to recover from interpretation failures and empirically evaluate them in a user study.

\subsection{Dialogue Systems to Support HRI}

Research on natural-language interaction with robots has a long history. The earliest approaches studied medical $[109,116]$ and office assistant $[2,4]$ domains. Research over the past two decades has focused on human-robot dialogue in navigation $[79,97,103,108,114]$ and manipulation [36, $46,64]$ tasks, but many other domains exist [15, 35, 86]. A more comprehensive overview of the subject can be found in reviews by Kruijff [55] and Mavridis [76].

Natural-language dialogue enables robots to ask clarification questions [30, 59, 84] and provide status updates $[60,115]$ to conversational partners. Some of this research contributes to disambiguation methodologies [37, 56, 121], but a few attempt to renegotiate natural-language instructions that are not possible to execute $[24,77,90]$. In comparison, TeamTalk uses a data-driven approach to handle referential ambiguity and impossible tasks. Furthermore, we focus on identifying properties of the surroundings that robots can leverage to resolve problematic instructions.

\subsection{Error Detection in Dialogue Systems}

Several groups have developed miscommunication detection methods for spoken-dialogue systems. Commonly, confidence values produced by automatic speech recognition (ASR) can determine whether the system correctly interpreted a user's utterance. These approaches have primarily been studied at the level of phonemes, words, and whole utterances [6, 25, 29]. Alternatively, San-Segundo et al. [96] used information from the language model and parser to train a neural network-based classifier that detected misrecognized words and out-of-scope phrases. Walker et al. [120] evaluated many features of the natural-language understanding (NLU) and dialogue management components of a telephony-based dialogue system to detect misunderstandings and other errors. Bohus and Rudnicky [13] studied ways to combine evidence from the speech recognizer, parser, and dialogue manager to estimate confidence about an input utterance. Litman et al. [63] considered prosodic features for detecting ASR errors. More recently, Meena et al. [78] explored using a range of features from ASR, NLU, and natural-language generation system logs to automatically detect miscommunication in online and offline settings.

Detecting and diagnosing errors in spoken-dialogue systems has received a considerable amount of attention and has led to established processes. However, most of these detection approaches rely on evidence from the spoken-language input and not from other sensory data. Moreover, these approaches largely operate in nonsituated domains. Situated grounding problems have not received this level of attention, but the success of ASR and NLU approaches could be extended by including path planner and environment information.

\subsection{Error Recovery in Dialogue Systems}

Understanding human error recovery strategies usually starts by conducting Wizard-of-Oz experiments in which naïve participants encounter simulated ASR problems while interacting with a dialogue system controlled by human wizards. Several strategies exist for repairing communication breakdowns. Zollo [123] found that human wizards used strategies that systems at the time 
were not capable of using, such as prompting users about a single word or phrase. Other strategies include explicit and implicit confirmations, asking to repeat a word or phrase, clarification requests about ambiguities, and disregarding any information under discussion that is not crucial to the dialogue task [98]. Skantze [105] found that human wizards acting as systems in a map navigation domain avoided explicitly declaring a non-understanding and instead opted to move the dialogue forward by asking alternative questions related to the task. Bohus and Rudnicky [14] showed that dialogue systems can learn the same strategies.

Recovery strategies for misunderstandings in dialogue systems boil down to explicit and implicit confirmation strategies. These two recovery strategy types are common in nearly all spokendialogue systems capable of error handling [17]. There is also general agreement about the pros and cons of both strategies [10]. Explicit confirmations allow the system to constrain the user response to a confirmation (e.g., "yes" or "no") but also require an additional dialogue turn to complete. Implicit confirmations present the system's understanding of a user utterance and provide the user the option to interrupt the system if there is a misunderstanding. Without user intervention, the system will assume that its interpretation is correct for implicit confirmations. Present-day dialogue systems are better equipped to handle explicit confirmations because their responses are easier to predict than responses to implicit confirmations. Krahmer et al. [52] conducted an extensive analysis of explicit and implicit confirmations. They identified positive and negative signals that users made when the dialogue system used these strategies, and were able to automatically detect dialogue system errors based on these cues.

Recovery strategies for non-understandings operate at a coarser level than misunderstandings because the system interprets little to no part of the user's utterance. Dialogue systems typically indicate a failure to understand and ask the user to repeat [10]. However, there have been attempts to better diagnose non-understandings. For example, Gorrell et al. [39] attempt to better understand the type of problem by classifying any known information from the non-understanding into one of a set of help options. Those help options attempt to give the user more feedback on how to speak to the system given results from a domain classifier (e.g., a when versus where question). System developers of a large-scale bus information dialogue system suggested a method for better diagnosing non-understandings by extracting words from the non-understood input and giving the user alternative ways to answer questions that the system could anticipate [91].

Bohus [10] offers several human-computer recovery strategies for non-understandings. The simplest strategies include lexically entraining users to words and phrases that the system can interpret, offering a help option, using alternative methods of input such as DTMF (touch-tone), and backing off to system-directed control. Other strategies from his work include proposing an alternate task-related question, asking for a short answer, providing an example of what the system can understand (e.g., "You can say yes or no"), and offering advice on how to speak to the system (e.g., more slowly and softly). Skantze [106] also investigated methods for overcoming errors in spoken dialogue found in human-human communication.

Dialogue system strategies primarily focus on recovering from errors related to spokenlanguage interpretation. Handling speech-recognition errors is the most common task. In this article, we study error-recovery strategies for communication problems in situated dialogue, which occur even when the user's speech is interpreted correctly.

\section{RESEARCH PLATFORM: TEAMTALK WITH USARSIM}

In this section, we describe the architecture of the TeamTalk spoken-dialogue platform used in this work. To enable communication with virtual robots, we also discuss the USARSim simulator. 


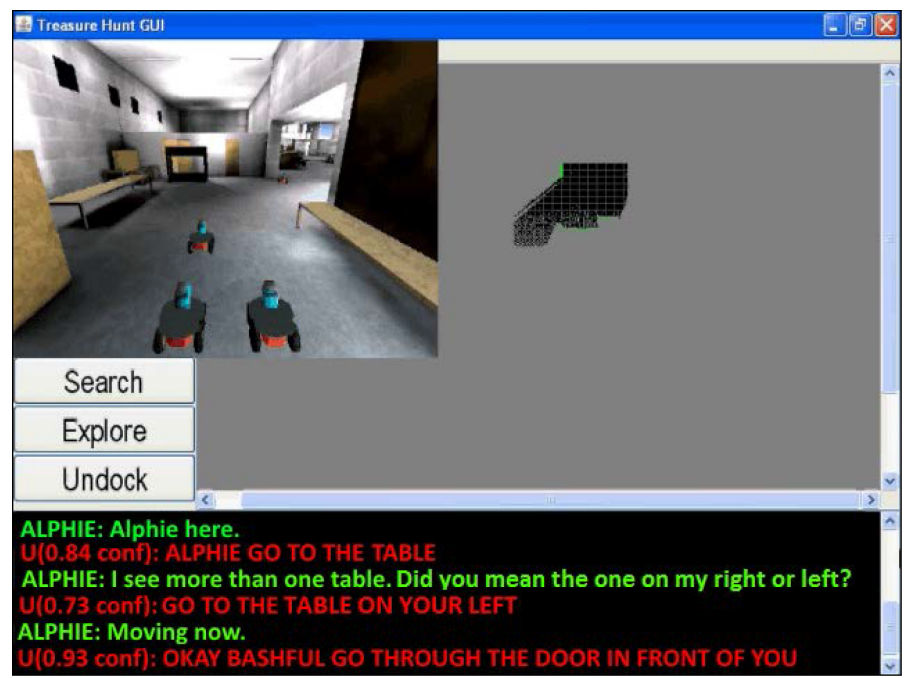

Fig. 2. The TeamTalk graphical user interface displaying a 3D virtual environment with a team of robots (left) and map (right). The dialogue history is displayed in the bottom of the window.

\subsection{TeamTalk Human-Robot Dialogue Platform}

TeamTalk ${ }^{2}$ is a platform for spoken-dialogue interaction in human-robot teams situated in realworld and virtual spaces $[43,68]$. It provides a spoken-language interface between one human operator and a team of one or more robots. TeamTalk encompasses over a decade of research and development in task-oriented dialogue for the search-and-rescue and navigation domains. Commands can be issued to an individual robot or a team of robots simultaneously. The work presented in this article extends TeamTalk with capabilities that enable execution of a wide range of situated tasks, with improved detection and recovery of situated grounding problems.

The TeamTalk virtual platform features two views, a live map and a 3D view of the environment, with models for robots and other objects. The graphical user interface (GUI) displays the current location of robots and the areas that they have traversed, as shown in Figure 2. The map information is displayed as a two-dimensional occupancy grid that continually updates as the robots navigate. TeamTalk supports discrete movement and rotation commands used in motion planning.

With TeamTalk, users issue navigation instructions. Commands to TeamTalk include movements of specific distances (e.g., "Move forward five meters") and movements to objects defined in the robot's ontology (e.g., "Go to the box"). Users can also specify rotations (e.g., "Turn right"). TeamTalk also supports naming locations (e.g., "Name this location Alpha") and querying the whereabouts of robots in the environment.

\subsection{TeamTalk Software Architecture}

Figure 3 shows an overview of the TeamTalk architecture configured for a two-robot team. TeamTalk features a pipeline architecture that incorporates a layer responsible for maintaining a dialogue with the user (human interaction layer) and a layer responsible for robot control (robotics layer) [68].

\footnotetext{
${ }^{2}$ Source code and data: http://trac.speech.cs.cmu.edu/repos/teamtalk/branches/tt-olympus; documentation is available at http://wiki.speech.cs.cmu.edu/teamtalk.
} 


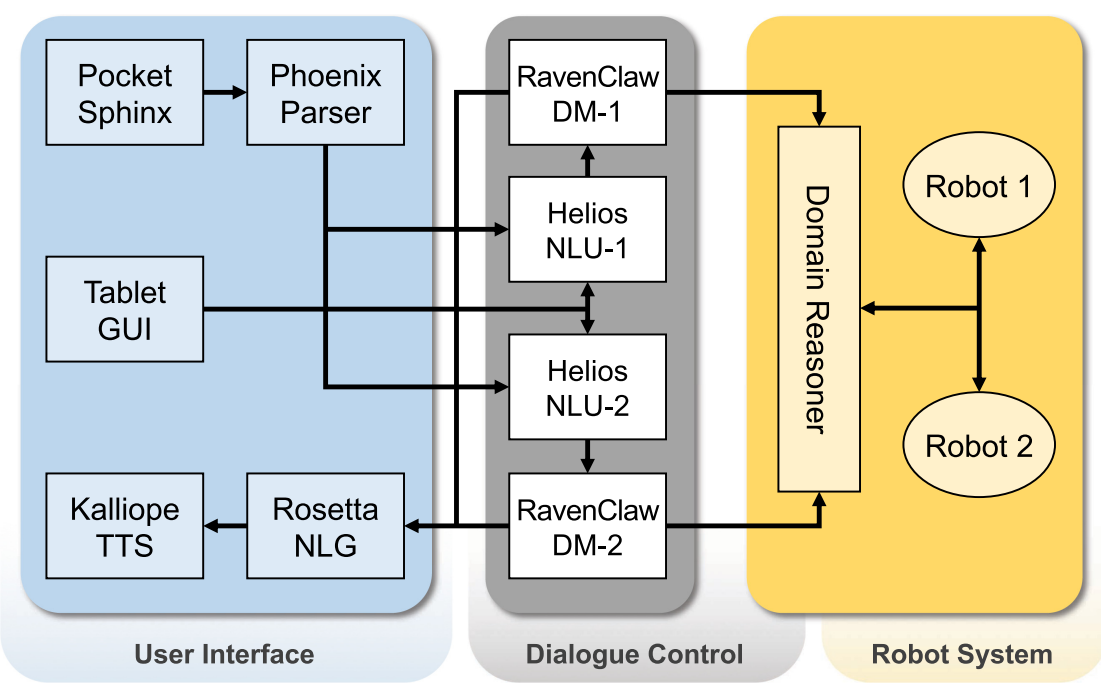

Fig. 3. Overview of the TeamTalk architecture [68].

4.2.1 Human Interaction Layer. The front end for TeamTalk handles user input and displays the robots' shared map. Optionally, a separate window can display the 3D rendering of robots in the virtual environment. The GUI also displays the robot location and the recent conversation history (six lines of dialogue). Commands may be issued by speaking into a microphone or typing directly into the GUI. Robots respond with synthesized speech.

The human interface component is built using the Olympus ${ }^{3}$ Spoken Dialogue Framework [12]. Olympus provides all of the software components necessary for a task-based dialogue system. Among the components are those to manage the state of the dialogue (the RavenClaw dialogue manager [14]), record and decode speech (the PocketSphinx speech recognition engine [47]), parse the input to extract its semantics (Phoenix), annotate speech and parsed input for confidence (Helios), generate language (Rosetta), and produce synthesized speech (Kalliope). Communication between components is supported by the Galaxy Communicator message-passing infrastructure [8].

4.2.2 Robotics Layer. TeamTalk's domain reasoner communicates with the robot hardware (real or virtual). When the dialogue manager decides on the course of action that the robot should take, TeamTalk passes that information to the domain reasoner. Tasking could originate from usergenerated instructions or responses to clarification questions. Messages are then sent to a robot's path planner as absolute (GOTO $(x, y, \theta)$ ) or relative (MOVEREL (translation $t, \theta)$ ) moves. In our study of situated grounding problems, all communications are between a human operator and a single robot.

\subsection{USARSim and MOAST}

TeamTalk supports spoken dialogue with virtual robots using USARSim ${ }^{4}$ [22], a high-fidelity simulator designed for conducting research with mobile robots. It supports the rendering of virtual environments and robots using the Unreal Engine.

\footnotetext{
${ }^{3}$ Source code: http://trac.speech.cs.cmu.edu/repos/olympus. Documentation: http://wiki.speech.cs.cmu.edu/olympus.

${ }^{4}$ Source code: http://sourceforge.net/projects/usarsim.
} 
In TeamTalk's virtual configuration, robots are simulated using the MOAST SIMware software [5]. MOAST is tightly integrated with USARSim, as it serves as a proxy for robotics middleware. In this work, the robot uses an $A^{*}$ path planner that references locally generated mapping data. A MOAST robot enters a virtual environment by spawning as a process, joining the virtual environment, and establishing communications with TeamTalk. The occupancy grid map starts out empty and expands as a MOAST robot traverses the environment.

\section{DETECTING GROUNDING PROBLEMS WITH THE TEAMTALK SITUATED REASONER}

Without situated reasoning, TeamTalk as a dialogue platform allows robots to move a specified distance or to a predefined labeled object. The TeamTalk Situated Reasoner (TSR) is an extension that provides a robot skills useful for engaging in physically situated dialogue (as described in Section 2.3). Moreover, TSR combines these skills while managing a representation of environment context. TSR includes algorithms for egocentric spatial reasoning, proximity reasoning, and field of view. Situated grounding problems such as referential ambiguity and impossible tasks are detected by leveraging these skills. Together, this functionality expands the range of tasks that a robot can do with TeamTalk.

\subsection{Overview}

TSR extends TeamTalk's domain reasoning in order to expand robots' capabilities in physically situated contexts. Figure 4 presents TSR as it references static and dynamic contextual information, described in more detail in Section 5.2.2. TSR detects situated grounding problems in the robot navigation domain (e.g., ambiguous requests such as "Go to the door" when there are multiple doors and impossible-to-execute ones such as "Go through the door" when there are no open doors). When TSR detects a problem, it sends the diagnosis back through the dialogue manager so that it can be presented to the user.

One of TSR's primary contributions is connecting dialogue actions to spatial relationships between a robot and objects in the environment. For example, given the robot's current position in the environment and its relative rotation to North, TSR determines whether a given landmark is "in front," "behind," "to the right," or "to the left" of the robot. It computes the total relative rotation that the robot must make to face a given landmark and calculates the spatial relationship. TSR also reasons about proximity between objects (e.g., identifying "box near the bed" in the environment) and between objects and the robot (e.g., "box near you"). Proximity handling is achieved via a backoff model that uses estimates of Hall's [40] interpersonal distances: it first checks for nearby objects within a personal space $(\approx 1 \mathrm{~m})$ of each other, followed by those within a social space $(\approx 2 \mathrm{~m})$ based on the number of matches available in the current situation.

\subsection{Representing Context in Physically Situated Dialogue}

TSR connects spoken language with robot mapping and path planner information. While some context can be represented statically and retrieved from a structured knowledge base, there is also dynamic information that must be computed in real time.

5.2.1 Language Context. Language context consists of all of the information extracted from the user's speech. TSR receives language context as user input and processes it for relevance in the current situation. Features from the language context include information about recognized speech, semantics obtained from the language, and a confidence score. Determining referable objects requires that they be present in the speech recognizer's vocabulary and that there is a correspondence between words and the physical environment (i.e., symbol grounding). Following speech 


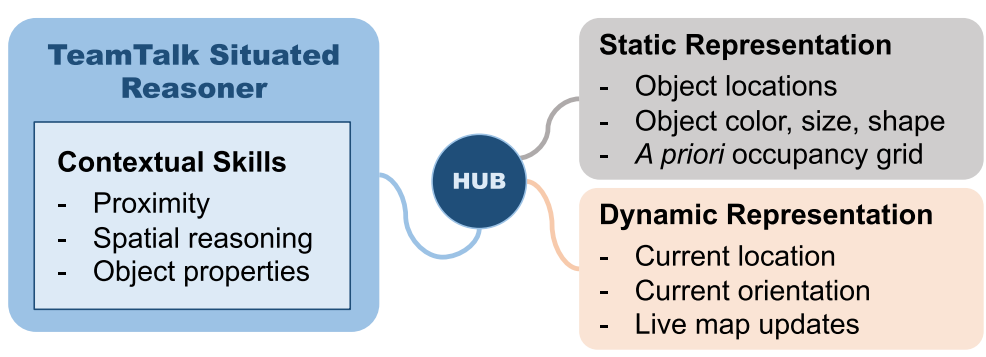

Fig. 4. The TeamTalk Situated Reasoner (TSR) combines static and dynamic information to detect situated grounding problems.

recognition, the parser produces a semantic representation. The dialogue manager then determines relevant responses by selecting dialogue actions. The dialogue actions that accept object terms as parameters define tasks in which those objects can be used. The grounding process then binds recognized words to dialogue manager concepts. For example, given the utterance "Move to the box," the dialogue manager will bind the object term "box" to the concept landmark for the dialogue action associated with moving to objects.

5.2.2 Physically Situated Context. Physically situated context is collected from the robot's knowledge base, map, and path planner. We consider two types, static and dynamic context. Static context contains observable properties of the surroundings that do not change, while dynamic context may change as the robot moves.

Context awareness for robot navigation is a function of available sensor data and relevant processing. In this article, we use the following sources of context defined in previous work [56]: (1) occupancy, (2) proximity, (3) orientation, (4) color, and (5) geometry. These sources have achieved strong levels of automatic performance with state-of-the-art localization and perception software $[21,93]$. They can also be used to distinguish objects when clarifying referents with the user. Occupancy is awareness about the positions of objects, walls, obstacles, and space. This requires reasoning about space and where space is occupied. Proximity describes how close the robot is to each object in the environment. Proximity requires reasoning over position (the $(x, y)$ coordinates of dialogue partners and objects in the environment) and distance (how close or far an object is from the robot or other objects). Understanding spatial terms requires access to the robot's current position and how surrounding objects represent spaces near the front, back, left, or right sides of the robot. Orientation provides information about the objects that the robot is facing and the other objects that each object is facing. This requires reasoning about spatial terms such as "left" and "right." Color can be used as a visual property to distinguish possible referents by using color names, such as "blue" or "red." Finally, geometry awareness requires reasoning about the shape and size of objects. In our representation, both color and geometry are encoded in the robot's environment and are labeled $a$ priori.

Static Context. TSR stores static context in a knowledge base using a structured ontology, retrievable by queries [95]. We use an instance of the OWL representation of the Protégé ontology [49] developed for TeamTalk. Protégé is an open-source ontology platform that has seen wide adoption in artificial intelligence research. This representation allows the robot to store past actions and the locations of objects. The interaction history is stored as episodes that represent time-aligned sequences of dialogue commands and corresponding actions taken by the robot. We extended the ontology in this work to represent physical situations by adding object properties and proximal relations between objects. 


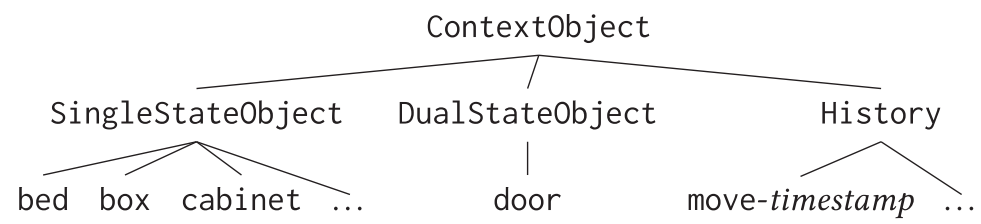

Fig. 5. Ontology classes in the robot navigation domain.

The robot's environment is represented in the ontology as a semantic map. The map contains instances of defined object types. We use the presence of objects in the robot's semantic map to detect situated grounding problems. For example, when the user mentions a "door" landmark, TSR tabulates all visible doors in the surroundings. The locations of these objects are used to calculate spatial and proximal relations between objects. The robot also refers to objects in the semantic map when initiating recovery strategies. In the case of the door example above, for a referential ambiguity, TSR would retrieve the possible referents and describe them in a way that uniquely distinguishes them (e.g., accessing color in the form of the clarification, "Did you mean the blue door or the brown door?").

We defined the robot's assumed capabilities by examining the SCARE corpus [110] of humanhuman dialogue, which used the direction-giver-follower paradigm mentioned in Section 2.1. We identified the properties of objects referred to by interlocutors, paying particular attention to occurrences of the direction-follower asking for clarification (similar to the robot's role in our setup). After review of the corpus, we populated the ontology with static information, including (1) object name, (2) object coordinates, (3) object region, (4) object color, (5) object shape, (6) object size, (7) nearby objects, (8) object state (i.e., whether objects such as doors are open or closed), (9) a visibility flag indicating whether the robot has seen the object, and (10) dialogue history. The ontology uses the classes presented in Figure 5 for objects in the robot's environment and past interactions in the robot's history. Instances represent individual objects and populate the classes representing basic object types, such as bed or door. They also inherit the properties defined for that object class.

Walls do not change in the robot's environment. This assumption allows us to define an additional type of static context, an a priori occupancy grid that the robot can use for grounding plans. An occupancy grid assigns a binary value to each point in order to indicate whether it is occupied or open space. We used Lester's implementation of $A^{*}$ pathfinding [61] to process the grid in real time. When the robot receives an unambiguous navigation command, the $A^{*}$ pathfinding algorithm checks to see if a path is possible to the candidate goal destination. When there is no possible path, the robot detects this as a type of impossible-to-execute instruction.

Other ontologies exist that can support situated natural-language interaction. KnowRob [113] has been shown to support reasoning and knowledge acquisition for robots doing manipulation tasks, and has been used to support reference resolution for robotics applications [122]. ORO [58], also implemented with Protégé, supports common-sense reasoning for HRI.

Dynamic Context. Physically situated grounding involves processing dynamic context; this consists of (1) spatial relations between the robot and other objects, (2) proximity relations between objects, and (3) the inference of proximity between the robot and other objects. TSR uses the robot's current position, orientation, and live map to compute dynamic context. As the robot moves in the environment, streaming sensory input causes this information to change. Whenever the user issues a request that relies on dynamic context, it is recomputed in real time.

TSR collects live occupancy grid data to enable autonomous obstacle avoidance strategies. In simulation, we use a MOAST $\mathrm{A}^{*}$ path planner that operates over streaming mapping data (this 


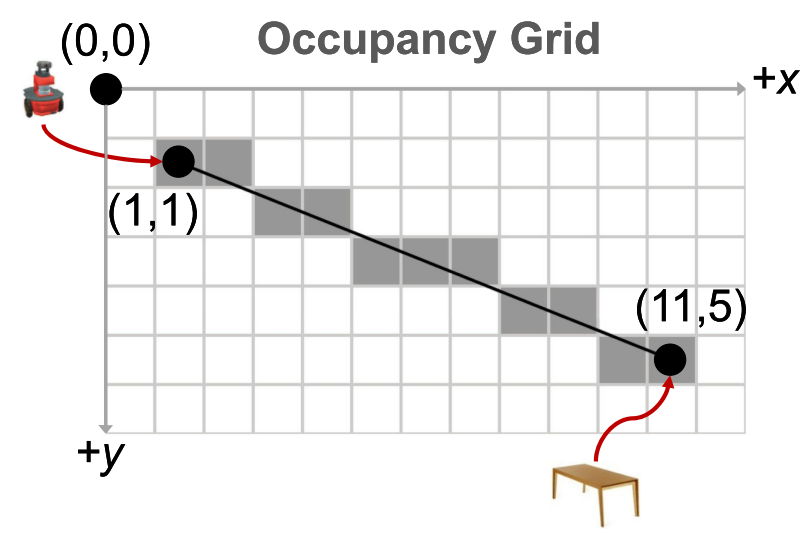

Fig. 6. TSR calculates field of view between the robot and referents using Bresenham's line algorithm [18].

path planner is separate from the $\mathrm{A}^{*}$ pathfinding algorithm that uses the a priori occupancy grid). This dynamic path planner uses position information to find a short path to goal locations (i.e., $(x, y)$ points in the robot's map). The robot uses MOAST obstacle avoidance libraries to prevent collisions with objects. Thus, initially possible tasks may become impossible whenever the robot finds no possible path because of a newly observed obstacle.

5.2.3 Field of View. TSR keeps track of the objects that the robot has encountered using a visibility property. Field of view indicates which objects the robot can and cannot see. We accomplish this using a combination of constraints applied to the robot's surroundings. Before the robot enters an environment, all objects are marked as not visible. Whenever the robot changes its position, we recalculate object visibility using Bresenham's line algorithm [18] between the robot and the $(x, y)$ locations of all surrounding objects. Whenever the robot calculates its line of sight between itself and an object, if a wall blocks it (calculated using the a priori occupancy grid), the object's entry remains marked as not visible. Otherwise, the object is marked visible. Figure 6 illustrates Bresenham's line algorithm being calculated between the robot and a table in its surroundings.

\subsection{Task-Independent Skills}

TSR includes two task-independent skills: (1) referent count detection and (2) open-endedness detection. For (1), the number of possible referent candidates that match the object term in an instruction determines the general situated grounding problem regardless of domain. If there are zero matches for a referent, the instruction is impossible to execute. If there is more than one match, the instruction is considered referentially ambiguous.

For (2), actions are defined for the dialogue manager as requiring specific parameters to be executable. TeamTalk can prompt the user for any missing information if it is needed to complete an action. For example, if the user indicates a move without specifying a distance or referent, TeamTalk will prompt for the missing information.

\subsection{Physically Situated Skills}

Physically situated skills rely on static and dynamic information. Given (1) a command that references an object and (2) a search space of objects from the robot's knowledge base that match a basic object type, we narrow the search space if a spatial relation, proximity relation, or intrinsic property is provided. In this section, we describe skills for processing egocentric spatial relations, proximity relations, and intrinsic property relations. 


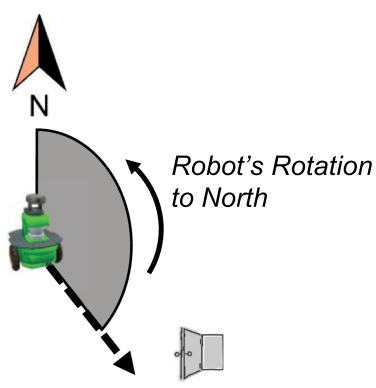

(a)

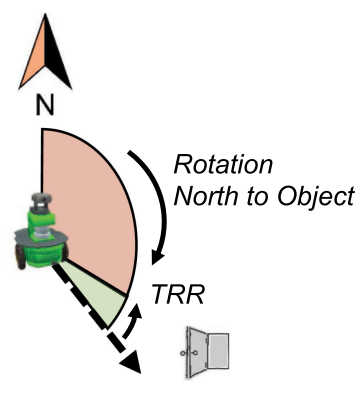

(b)

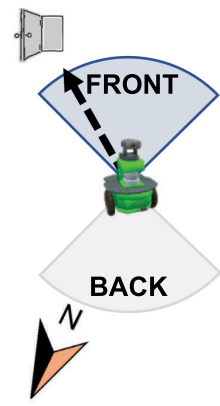

(c)

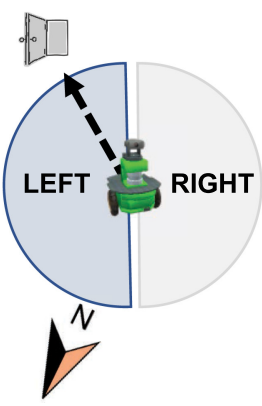

(d)

Fig. 7. TSR determines egocentric spatial relations by calculating the total relative rotation (TRR) between the robot and a referent (in this case, a door) using the cardinal direction North as a global point of reference. The algorithm finds (a) the robot's rotation relative to North and (b) the subsequent rotation to the referent, resulting in the calculation of the total relative rotation between the robot and the referent. Here, the robot determines the referent to be (c) in front and (d) to its left using spatial thresholds defined for the total relative rotation value.

5.4.1 Egocentric Spatial Relations. TSR interprets spatial information by calculating egocentric proximity relative to surrounding objects using the robot's real-time position. Figure 7 shows how TSR determines egocentric spatial relations between the robot and a referent. TSR tracks the robot's $(x, y)$ position, the $(x, y)$ positions of all matching referents, and the angle $\theta$ representing the rotation of the robot relative to the cardinal direction North. With this information, TSR calculates the robot's total relative rotation (TRR), the amount of rotation that the robot would need to rotate to face each referent.

TSR calculates the rotation from the robot's current position to the referent using thresholds to determine spatial relations. In our studies, we configured the thresholds with the prototypical relations shown in Figures 7(c) and 7(d). For instance, if the instruction is "Move to the box on the right," TSR first retrieves all visible boxes in its static knowledge base. It then restricts the list to only those on the robot's right by computing TRR.

This approach supports both spatial interpretation and spatial description. For interpretation, TSR checks if the robot's TRR results in a spatial relation that matches a part of an instruction (e.g., "box in front of you"). To reduce the occurrence of unintended impossible instructions, during interpretation, TSR attempts to find all valid spatial relations between the robot and a referent. For instance, if the robot is both in front and to the right of a referent, a mention of either a front or right relation would match. On the other hand, when a description must be generated about a referent that uses its egocentric proximity, such as when prompting the user for clarification, TSR uses a maximally restrictive distinction.

5.4.2 Proximity of Surrounding Objects. TSR calculates proximal relations for objects near referents. For interpretation, given a mention of a referent using its relationship to an object in close proximity, TSR checks whether a corresponding match exists in the surroundings. We assume a hierarchy of proximity, in which relations are first checked against Hall's definition of personal distance and then Hall's definition of social distance [40]. For example: "Go to the box near the bed" changes to the task of finding all boxes near beds, where box=referent; bed=proximity_object. The robot uses the $(x, y)$ positions of referent matches and objects that match the proximity relation in the environment to determine nearness relations. 


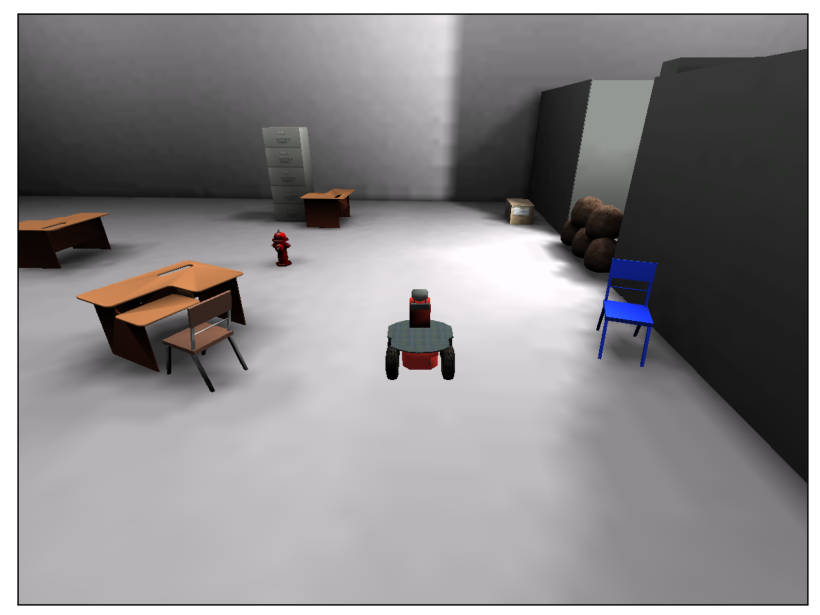

Fig. 8. An example situation used in evaluating TSR with the route instruction "Go to the chair" (a case of referential ambiguity showing two possible chairs). The chairs have different colors and, given the robot's current position, are directly to the left and right of it.

5.4.3 Intrinsic Object Properties. Intrinsic object properties are each object's basic object type, color, size, and shape. When TSR receives a reference to an object that mentions an intrinsic property (e.g., "black lamp"), it checks to see if there is a complete match in the ontology. If the reference mentions an intrinsic property that does not match any entries in the robot's ontology, any action associated with the command is considered impossible-to-execute.

\subsection{Evaluation}

To evaluate TSR, we used a corpus of 50 commands given to a simulated robot. We also developed scenarios corresponding to each instruction using 10 different virtual environments developed with the UnrealEd game map editor. In a related study, these same scenarios were used to crowdsource recovery strategies for how a robot should handle situated grounding problems [73]. Each scenario defined the information known to the robot, its current position in the environment, and a route instruction as input. Figure 8 presents an example scenario of referential ambiguity.

The scenarios were constructed to balance the presence of situated grounding problems and the types of context available to the robot. Twenty-five (50\%) of the scenes depicted referential ambiguity, 15 (30\%) depicted impossible situations, and 10 (20\%) were executable. We included more referential ambiguity examples because we anticipated more variation in strategies than other problem types. Ambiguous situations included variations of egocentric spatial relations, proximity of surrounding objects, intrinsic object properties (color or size), and history ${ }^{5}$ (referring to previously mentioned objects). The number of context sources available was evenly distributed across five scene categories: one with all four sources of information and three that included all but one source (e.g., one scene type used intrinsic properties, history, and egocentric spatial relations but excluded proximity). The impossible situations were divided into two categories: the route instruction could not match to a referent in the environment (e.g., the referent was not present or the instruction overspecified possible referents) or the path to the goal was blocked. All scenarios can be found in [67].

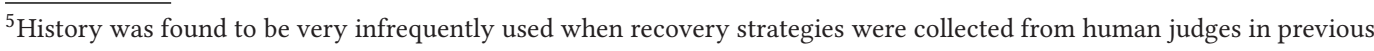
work [73]; thus, we focused on detecting other sources of information.
} 
Table 1. Results Comparing Performance at Correctly Detecting Situated Grounding Problems

\begin{tabular}{lcccc}
\hline Evaluation & Ambiguous & Impossible & Executable & Overall \\
\hline TeamTalk Baseline & $0 \%$ & $13 \%$ & $80 \%$ & $20 \%$ \\
TeamTalk with TSR & $\mathbf{1 0 0 \%}$ & $\mathbf{1 0 0 \%}$ & $\mathbf{1 0 0 \%}$ & $\mathbf{1 0 0 \%}$ \\
\hline Number of Trials & 25 & 15 & 10 & 50 \\
\hline
\end{tabular}

The focus of the evaluation was to assess how well TSR could detect situated grounding problems by giving the robot instructions while it was in several virtual environments. An ablative analysis compared the performance of TeamTalk with TSR compared to a baseline version of TeamTalk without TSR. Note that TeamTalk without TSR can go to locations of basic object type only (e.g., "box" but not "box on the right"); thus, any additional phrases included beyond the basic object type were ignored by the parser.

Results can be found in Table 1 . TeamTalk with TSR was able to correctly identify whether or not all scenarios had a problem. For the baseline system, only 10 of the 50 trials were correct. Of these 10 , the baseline system was able to do only 8 out of the 10 executable scenarios. Finally, the baseline system could detect two impossible scenarios simply because they referred to only a basic object type that was not present in the environment.

A failure analysis showed that the baseline version of TeamTalk could not detect problems primarily due to the lack of situated reasoning (i.e., reasoning capabilities to support spatial, proximal, and intrinsic property reasoning) and limitations of the parser. For the 25 failures associated with detecting referential ambiguity, all of them had the same issue in common: the path planner required a single, unique referent. The implementation would do nothing to move the robot if the environment contained more than one instance of a basic object type being referenced in an instruction. While they could all be properly parsed, no control logic existed to support referential ambiguity. The failures for detecting impossible situations with the baseline were more diverse. A failure analysis revealed that three instructions were not detected as impossible due to the lack of spatial reasoning, three due to the lack of proximal reasoning, and one due to the lack of intrinsic property reasoning. Four instructions were missed because the baseline version could not reason about object states (e.g., closed doors or objects that cannot be moved through when receiving "move through" instructions). Two instructions were not detected because they required interpreting messages from the path planner that a path was not possible. Of the impossible instructions, four also did not have sufficient parsing support for egocentric spatial relations, proximity, or intrinsic properties. The two failed executable instructions also required the same parsing support to have an unambiguous interpretation.

The results demonstrate that TSR can handle situated interaction in controlled settings. We note that the results are limited to commands that involve one action at a time, with support for one referent. More flexible natural-language instructions referring to the same intents would be possible with methods such as crowdsourcing instructions (e.g., as in [111]). Detecting situated grounding problems is the first step to resolving them; the results presented here provide a supportive foundation for initiating recovery strategies, described in the next section.

\section{RECOVERY STRATEGIES FOR PHYSICALLY SITUATED DIALOGUE}

Recovery strategies are actions that a dialogue partner can take to repair grounding problems. We first present an analysis of human-human navigation corpora, identifying where people following directions would initiate recovery strategies. We then describe recovery strategies that a robot could use to recover from situated grounding problems. 
Table 2. Recovery Strategy Types Observed in the Map Task and SCARE Corpora for Referential Ambiguity and Impossible-to-Execute Instructions between a Direction-Giver (DG) and Direction-Follower (DF)

\begin{tabular}{|c|c|c|}
\hline Problem Type & Strategy & Examples \\
\hline \multirow[t]{5}{*}{ Referential Ambiguity } & Confirming a Single & (DF) It's the one I'm closest to this one? \\
\hline & Referent & (SCARE, Dialogue S4) \\
\hline & & (DF) Next to the stairs? (SCARE, Dialogue S12) \\
\hline & & (DF) No so do you want me to go right round \\
\hline & & pine forest? (Map Task, Dialogue Q1EC4) \\
\hline \multirow[t]{2}{*}{ Referential Ambiguity } & List Options and & (DF) This one or the left? (SCARE, Dialogue S2) \\
\hline & Query & $\begin{array}{l}\text { (DF) OK, this door or that door? *points with } \\
\text { shared gaze }{ }^{*}(S C A R E \text {, Dialogue S3) }\end{array}$ \\
\hline \multirow[t]{3}{*}{ Impossible-to-Execute } & Propose Alternative & (DG) So ehm ehm I'm kind of stuck. \\
\hline & Plan & (DF, in reply) Can I just go straight to the \\
\hline & & saloon bar? (Map Task, Dialogue Q1EC3) \\
\hline
\end{tabular}

\subsection{Understanding Human Miscommunication Behaviors}

In order to understand the variety of strategies that people take when following route instructions, we examined existing human-human dialogue corpora in the navigation domain [72]. In these corpora, a pair of dialogue partners discussed navigation tasks. By convention, one person is assigned as a direction-giver (DG) and the other a direction-follower (DF). Either can ask questions of the other, but the DG issues instructions that the DF attempts to follow.

6.1.1 Recovery Strategies from Dialogue Corpora. We discuss miscommunication in two human-human dialogue corpora, the HCRC Map Task corpus and the SCARE corpus.

- HCRC Map Task Corpus. The HCRC Map Task corpus consists of 128 unscripted English human-human dialogues (64 participants) in the navigation domain [1,72]. Participants were tasked with verbally describing how to replicate a path on the DG's complete map in the DF's incomplete map. The maps were two-dimensional schematics of a fictional area, containing an assortment of landmarks. Participants were assigned to either the role of the DG or the DF.

- SCARE Corpus. The SCARE corpus (Situated Corpus with Annotated Referring Expressions) contains 15 English dialogues with DG-DF pairs [110]. In this corpus, the DG guides the DF through a virtual world (the DF controlled an avatar in the environment). The DG had an annotated schematic map of the scene and sat in front of a monitor that showed the DF's view of the environment (the DF was in another room). Partners shared information not only by spoken dialogue but also by monitoring the DF's gaze and position in the virtual world.

For both corpora, we focused our analysis on questions from DFs about route instructions. For their recovery strategies, DFs make reference to their own perspective (egocentric proximity) or location (proximity to self); that of proximal objects in the vicinity (proximity to object); or intrinsic properties of the object, such as shape or size (intrinsic object property). The primary strategy categories are as follows, with example exchanges in Table 2:

(1) Confirming a single referent: The DF asks a yes-no question confirming a single referent.

(2) Listing options and asking a question: The DF presents a list of options in the form of a confirmation question. 
Table 3. Recovery Strategies for Referentially Ambiguous Situations

\begin{tabular}{ll}
$\begin{array}{l}\text { Recovery Strategy for } \\
\text { Ambiguous Tasks }\end{array}$ & \multicolumn{1}{c}{ Example Dialogue } \\
\hline $\begin{array}{l}\text { Disambiguation by egocentric } \\
\text { spatial relation }\end{array}$ & $\begin{array}{l}\text { Human: Move to the box. } \\
\text { Robot: I see more than one box. Did you mean the box on my } \\
\text { right or the box on my left? }\end{array}$ \\
\hline Disambiguation by color & $\begin{array}{l}\text { Robot: I see more than one box. Did you mean the red box or } \\
\text { the blue box? }\end{array}$ \\
\hline $\begin{array}{l}\text { Disambiguation by proximity to } \\
\text { object }\end{array}$ & $\begin{array}{l}\text { Robot: I see more than one box. Did you mean the box near } \\
\text { the chair or the box by itself? }\end{array}$ \\
\hline Disambiguation by size & $\begin{array}{l}\text { Robot: I see more than one box. Did you mean the big box or } \\
\text { the small box? }\end{array}$ \\
\hline $\begin{array}{l}\text { Disambiguation by a } \\
\text { combination of above sources }\end{array}$ & $\begin{array}{l}\text { Human: Move to the door in front of you. } \\
\text { Robot: I see more than one door. Did you mean the brown } \\
\text { door in front of me or the blue door in front of me? }\end{array}$ \\
\hline
\end{tabular}

(3) Propose alternative plan: The DF has difficulty interpreting the initial instruction of the DG and suggests an alternative.

\subsection{Recovery Strategies for Robots}

A recovery strategy takes the form of either a display of understanding (i.e., an explicit statement by the robot conveying some aspect of common ground with the user), a question relating to the user's instruction, or both. We investigated two types of recovery strategies, those for disambiguating referents and those for describing, and potentially refashioning, impossible-to-execute instructions. We define these recovery strategies using two sources of information: (1) occurrences of recovery strategies in human-human corpora and (2) the contextual information available to the robot.

6.2.1 Recovery Strategies for Referential Ambiguity. Recovery strategies to resolve referential ambiguity arise when there are multiple possibilities for converting an instruction to a plan. TeamTalk can indicate this type of problem by informing the user that multiple plans are possible. An initial analysis of questions posed by DFs in the Map Task and SCARE corpora, along with a crowdsourced dataset of human-authored recovery strategies described in previous work [73], revealed several important reasoning processes. These processes-(1) egocentric spatial reasoning, (2) proximity reasoning, and (3) intrinsic property reasoning (e.g., basic object type, shape, size, and color)-allow robots to initiate recovery strategies for situated interaction. Table 3 presents the set of recovery strategies that we investigated for referential ambiguity.

6.2.2 Recovery Strategies for Impossible-to-Execute Situations. Recovery strategies to resolve impossible-to-execute instructions are invoked when there are no possibilities for converting an instruction to a plan. TeamTalk can communicate this type of problem by informing the user that no plans are possible. To resolve problems, the robot may either state what is wrong with the current instruction, propose an alternative plan, or both.

Using our analysis of the HCRC Map Task corpus and the crowdsourced dataset described in [73], we identified five recovery strategies that robots can use for impossible situations. Table 4 presents these strategies. For the propose alternative plan strategy, the robot uses a constraint relaxation algorithm (described in Section 6.2.3). The robot tries to remove a constraint associated 
Table 4. Recovery Strategies for Impossible-to-Execute Situations

\begin{tabular}{ll}
$\begin{array}{l}\text { Recovery Strategy for } \\
\text { Impossible Tasks }\end{array}$ & \multicolumn{1}{c}{ Example Dialogue } \\
\hline $\begin{array}{l}\text { Declare impossible due to } \\
\text { ontology mismatch }\end{array}$ & $\begin{array}{l}\text { Human: Go through the table. } \\
\text { Robot: I cannot go through that. }\end{array}$ \\
\hline $\begin{array}{l}\text { Propose alternative plan } \\
\text { Ruman: Go to the door on the left. } \\
\text { Robot: I don't see the door on the left. Choose from the } \\
\text { following: Door in front of me or door on my right. }\end{array}$ \\
\hline $\begin{array}{l}\text { Declare impossible due to object } \\
\text { state }\end{array}$ & $\begin{array}{l}\text { Human: Go through the door. } \\
\text { Robot: I cannot go through that. }\end{array}$ \\
\hline $\begin{array}{l}\text { Declare impossible due to } \\
\text { planning failure }\end{array}$ & $\begin{array}{l}\text { Human: Move to the cone. } \\
\text { Robot: I cannot find a plan to get there from my current } \\
\text { location. }\end{array}$ \\
\hline $\begin{array}{l}\text { Declare impossible due to } \\
\text { obstacle interference }\end{array}$ & $\begin{array}{l}\text { Human: Go to the table. } \\
\text { Robot: I tried but I could not find a plan to get there from my } \\
\text { current location. }\end{array}$ \\
\hline
\end{tabular}

with the referent (e.g., "brown" in "brown box"), then present alternatives. When there is more than one option upon refashioning, the robot will make use of its disambiguation strategies. For the declare object state strategy, the robot receives an action that in some situations (e.g., moving through a doorway) is possible but in others is not. The robot will declare the problem to be impossible because the state of the object does not align with the requested action.

The remaining impossible-to-execute recovery strategies rely on the robot's a priori and realtime mapping data. If the robot receives a request to move to a location that according to its $a$ priori occupancy grid is not possible to move to, it will declare the move impossible. This declare planning failure strategy is triggered by a failure response from the a priori $\mathrm{A}^{*}$ pathfinding algorithm described in Section 5.2. Finally, the declare obstacle interference strategy occurs onlyin real timev-v-when the robot is already executing a task-due to a locally-detected obstruction from the robot's path planner.

6.2.3 Refashioning an Overspecified Instruction. Any property that is used to describe an object, including the object type itself, places a constraint on the possible matches for an intended instruction. By removing these constraints, the robot can form alternate descriptions. Consider the scenario in which the user tells the robot to move to the "big blue box" but no such object exists in the environment. The choice of whether the robot should remove the "big," "blue," or "box" constraint depends on the context of the immediate situation. We use a refashioning method to identify alternative referents, which we describe below.

When an original referent description is overspecified, that is, when it does not resolve to at least one match, the description can be altered and checked against the robot's surroundings. We can view each word as a type of constraint with different costs to remove. This requires defining an initial state space of possible instantiations of adjustments to the original referent description. The adjustments themselves can serve as the actions of the state space. The initial state space contains all combinations of the original referent description. With the original referent description as the start state, we formally define the search space in Figure 9.

We use Forward $A^{*}$ Search to find the cheapest action to take in the state space of possible referent descriptions. An example state space for the referent "big blue box" can be found in Figure 10, in which entering a new state means making an adjustment to the original referent. Adjustments 


$\begin{array}{ll}\text { State } s \in S & \text { Some combination of words } s \text { based on the original referent description } \\ \text { Query } q & \text { Query to the knowledge base for the number of existing referents that match } s \\ \text { Start state } s_{0} \in S & \begin{array}{l}\text { Original referent description } \\ \text { Action } a \in A\end{array} \\ \text { Cost } c \in C & \text { The removal of words from the original referent description } \\ \text { Heuristic } h & \text { The number of removal actions to reach the current state }\end{array}$

Fig. 9. Search space for proposing alternative referents.
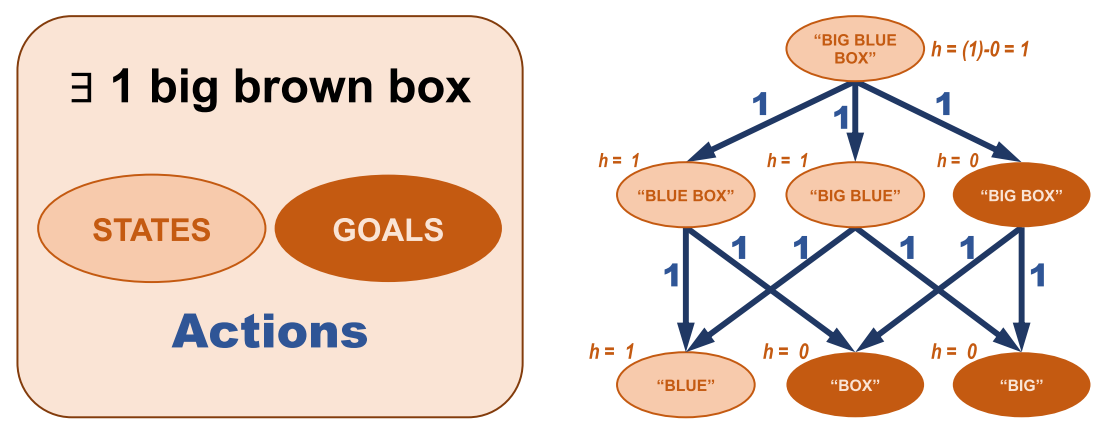

Fig. 10. Refashioning referent descriptions as a search space. Here, the referent "big blue box" is not possible, but the phrase is adjusted to "big box" to locate an alternative.

can be one of two types: removing an object property or relation (i.e., color, shape, egocentric spatial relation, or proximity to an object) or the basic object type. The basic object type is equivalent to the object category in the ontology (e.g., bed, box, cabinet, etc.). Each action has the same cost (1), but each state's heuristic value depends on the number of matches that exist for that referent description. After an adjustment, as long as the new referent description has at least one match, we include it in a propose alternative plan recovery strategy.

To improve performance, refashioning factors in the role of the word being removed from the referent description as part of the action cost. Using a cost assessment similar to the one proposed by Skantze [107], we rank actions by how significantly each possibility reduces the search space. This results in actions grouped by a search reduction cost value. Generally, we assign a higher cost to removing the basic object type than to an object property or relation: removing a basic object type generally opens the space of possibilities to a much larger one than object properties or relations. Additional details about the referent refashioning process can be found in [67].

6.2.4 Integration with the TeamTalk Architecture. Recovery strategies are implemented in TeamTalk's dialogue manager as Library Agents, a type of handler for domain-independent tasks. Library agents support the dialogue manager's error handling and can initiate sending status updates or clarification questions to the user from the situated reasoner, TSR. When TSR detects an instruction as requiring clarification, it passes a service call to the dialogue manager to present a recovery strategy to the user. For referential ambiguity, selection of a strategy requires TSR to compute relations that uniquely distinguish candidate referents. If there are multiple possible strategies, a heuristic or learned model can select appropriate content. For impossible situations, either a statement of the problem or list of alternatives can be presented; this depends on whether the original instruction can be refashioned into alternatives. After initiating a recovery strategy, TeamTalk then returns to a state of awaiting input so that the user can amend the original instruction. 


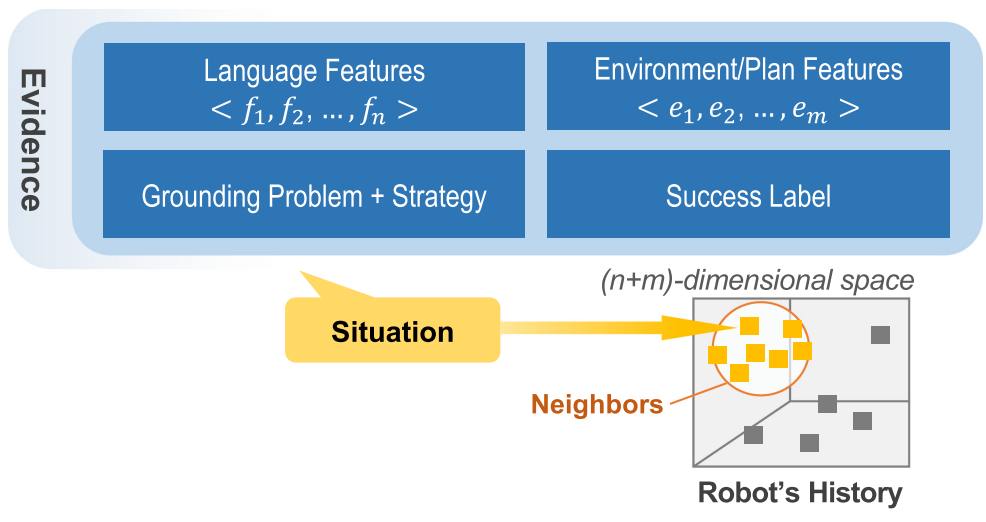

Fig. 11. With the $k$-nearest-neighbors learning algorithm, we cast a numeric representation of past dialogue recoveries as retrievable memories. The model will update the training data with new situations when they result in successful recovery. The closest successfully resolved situations to the current observation represent its neighbors. Circled are neighbors (pictured as yellow boxes) to the most recently observed situation.

\section{LEARNING TO IMPROVE RECOVERY STRATEGY DECISION-MAKING}

Dialogue systems can improve how they select recovery strategies over time using previous interactions. However, data collection with physically situated agents such as robots is difficult to parallelize; contextual features vary greatly based on the situation. Robots must build up an interaction history one situation at a time. The scale of data is therefore much smaller than what can be collected with mainstream dialogue systems such as those in commercial settings. We present an approach to improving recovery strategy selection using instance-based learning [82], in which the robot's previous successful recoveries serve as training examples. When the robot detects a situated grounding problem, it searches its history for similar situations to see how it resolved that problem in the past.

\subsection{Instance-Based Learning for Recovery Selection}

In order for the robot to learn to improve how it selects recovery strategies over time, we present the following classification task. Given a new observation of a situated grounding problem, the robot must select one of multiple possible recovery strategies. To initiate the learning process, we used a crowdsourced dataset collected previously [73]. New dialogues bootstrap the crowdsourced training data when the model selects a strategy that results in a successful recovery. As illustrated in Figure 11, the most common recovery strategy used among the nearest $k$ instances of similar situations is selected. Compared to many learning methods that train a model using data collected beforehand, a $k$-nearest-neighbors learning approach delays learning until the robot receives a new observation.

We developed a feature set that combines evidence from the robot's surroundings, the robot's plans (i.e., action sequences), and the content of spoken language commands that the robot receives. By bringing together these varied information sources, we expect that robots will better monitor situations and determine more effective strategies to recover as they make new observations. We identified several indicators that lead to grounding problems by examining dialogue corpora (i.e., HCRC Map Task and SCARE) and assessing the robot's capabilities. The feature list is summarized in Table 5.

7.1.1 Fast, Approximate Nearest Neighbors. Given a history of past interactions and a feature vector representing the current observation, we classify the situation and its grounding problem 
Table 5. Features for Numerically Representing Situations with the Instance-Based Learning Method $(\mathrm{C}=$ countable, $\mathrm{R}=$ real, $\mathrm{B}=$ Boolean $)$

\begin{tabular}{|c|c|c|c|}
\hline Indicator & Type & Number of Features & Feature Description \\
\hline \multicolumn{4}{|l|}{ Utterance Features } \\
\hline word_num & C & 1 & Count of words in utterance \\
\hline ref_prop_num & $\mathrm{C}$ & 1 & Count of object properties in the referent \\
\hline asr_conf & $\mathrm{R}$ & 1 & $\begin{array}{l}\text { Confidence score for top recognition } \\
\text { hypothesis }\end{array}$ \\
\hline parse_coverage & $\mathrm{R}$ & 1 & Ratio of uncovered to covered parse content \\
\hline num_parse_frag & $\mathrm{C}$ & 1 & Count of parse fragments \\
\hline \multicolumn{4}{|l|}{ Discourse Features } \\
\hline turn_number & $\mathrm{C}$ & 1 & Turn number within the dialogue session \\
\hline num_plan_matches & $\mathrm{C}$ & 1 & $\begin{array}{l}\text { Count of plans that match utterance: } \\
\text { executable }=1 \text {, impossible }=0 \\
\text { ambiguous } \geq 2\end{array}$ \\
\hline num_actions & C & 1 & Count of actions in utterance \\
\hline num_objects_instr & $\mathrm{C}$ & 1 & Count of objects in utterance \\
\hline impossible_step & $\mathrm{B}$ & 1 & True if utterance is detected as impossible \\
\hline \multicolumn{4}{|l|}{ Environment Features } \\
\hline distance & $\mathrm{R}$ & 2 & $\begin{array}{l}\text { Distance (meters) to (1) nearest match or (2) } \\
\text { goal }\end{array}$ \\
\hline num_matching_obj_instr & $\mathrm{C}$ & 1 & $\begin{array}{l}\text { Count of visible objects that match the } \\
\text { referent }\end{array}$ \\
\hline num_matching_obj_property & $\mathrm{C}$ & 21 & $\begin{array}{l}\text { Count of matching objects that also match } \\
\text { one of } 4 \text { spatial orientations, } 9 \text { colors, or } 8 \\
\text { shapes }\end{array}$ \\
\hline num_obj_property & $\mathrm{C}$ & 21 & $\begin{array}{l}\text { Count of visible objects that match one of } 4 \\
\text { spatial orientations, } 9 \text { colors, or } 8 \text { shapes }\end{array}$ \\
\hline num_obj_near_obj & $\mathrm{C}$ & 1 & Count of objects near other objects \\
\hline num_obj_near_matching_obj & $\mathrm{C}$ & 1 & $\begin{array}{l}\text { Count of objects near matching visible } \\
\text { objects }\end{array}$ \\
\hline num_nearby_doors & $\mathrm{C}$ & 1 & Count of doors nearby the robot \\
\hline
\end{tabular}

into one of a set of recovery strategies. We then find the most similar past situations (i.e., nearest neighbors) that resulted in success. For those neighbors to the current situation, we tabulate the recovery strategies used. We check to see if these neighbors used recovery strategies that are applicable. This reasoning happens directly after a grounding problem is detected and requires monitoring the current context stored in the robot's representation of the world, both dynamic and static.

We use the $k$-nearest neighbors $(k-\mathrm{NN})$ classification algorithm, which has proven to be effective for a variety of problem domains where underlying densities are not known [41]. Numerically representing situations in the physical space demands tracking many features. Since our data has a high number of dimensions, the traditional $k$-NN algorithm can be too slow as the amount of data collected increases. Instead, we used the FLANN toolkit: a fast, approximate approach that scales and does not impact response time to real-time dialogue [83].

Given the high cost of collecting physically situated interactions, the $k$-NN approach enables every new learned situation to be added to the training data as soon as the robot determines success or failure. In situations where the robot must select a recovery strategy, the user's verbal responses to its selection form a kind of implicit labeling. Dialogue exchanges where the robot 
detects a situated grounding problem, initiates a recovery strategy, and then grounds a single referent by the user's verbal response are labeled successful; all other recovery exchanges are labeled unsuccessful. We believe this is an effective labeling strategy as it leverages the knowledge of someone present in the context.

7.1.2 Bootstrapping with Crowdsourced Data. The initial training dataset consisted of a subset of recovery strategies collected from a crowdsourced study [73]. These were typed; thus, an annotator generalized them by labeling the responses with which properties they contained. Given a recovery strategy such as "Did you mean the blue or brown box?" the class label would be INTRINSIC: COLOR. The class types were intrinsic (color), intrinsic (size), object proximity, and egocentric spatial relation.

The crowdsourced study had 15 participants provide recovery strategies for each of 50 different situations. As such, the training data consisted of 15 identical feature vectors for each situation except that the class label varied with each human judge's recovery strategy. In total, this resulted in 228 training data entries for the 25 ambiguous situations and 49 training data entries for 11 impossible situations. Stratified subsets containing a third of each of the ambiguous and impossible groupings were withheld as test sets for the evaluation reported in Section 9.

For our data, we arrived at a value of $k=7$ after experimenting with odd values of $k$ until we observed diminishing performance on a development set, measured by neighbor agreement on classification. The learning algorithm expands the search when there is a tie among classes or only a contextually inappropriate classification is found, such as when the selected strategy does not fit the situation. In these cases, the value of $k$ is increased to the next-highest odd number and repeats.

\section{REAL-TIME EXPERIMENTATION WITH RECOVERY LEARNING}

We conducted a user study to better understand how dialogue systems for robots might recover from errors that occur over the course of a back-and-forth dialogue. The focus was to evaluate the $k$-NN learning algorithm by observing how the robot's selection of recovery strategies changes as it accumulated examples in real time. Six participants were recruited for the study. Each participant interacted with a robot using TeamTalk for six sessions. The longitudinal study consisted of dialogues between participants giving directions and an autonomous virtual robot (represented on a computer screen) following those directions; that is, no wizards controlled the robot's behavior.

\subsection{Method}

In this user study, participants viewed a four-wheeled ground robot (Pioneer P2AT) in a virtual environment (Figure 12). They interacted with the robot by speaking into a headset microphone. The experimenter provided the participant a task list of goal destinations to explore with the robot.

The user interface displayed a third-person view from behind the robot. In order to align with experiment goals, the robot's internal map was different from what appeared on the user interface. This difference in environment understanding resulted in controlled miscommunication between the participant and robot. The robot responded with feedback and clarification questions using synthesized speech.

8.1.1 Procedure. Participants were first greeted by an experimenter who explained the task, virtual robot, and user interface. The primary task was to conduct reconnaissance by exploring environments with limited and possibly outdated information. The robot would do the exploration while the participant issued tasks as a remote teammate. Participants received a paper map that provided an overview of the area, but they were informed that the robot may have the same map as the participant or a different one. The map also had a list of trials for which participants needed to give an instruction to the robot that included a keyword describing the target referent. Participants 


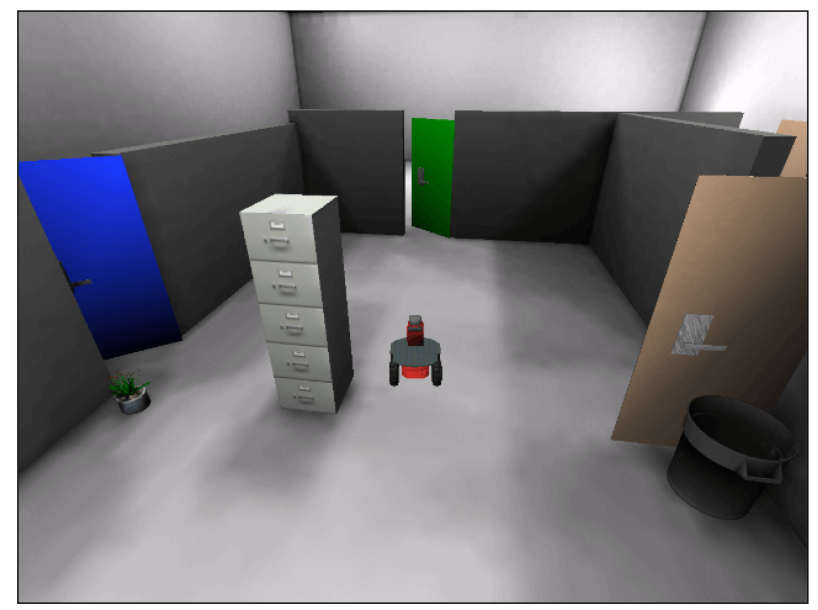

Fig. 12. An example referential ambiguity trial. Participants were tasked with using an underspecified referent ("door") in their instructions to the robot (centered).

needed to verify that objects in the trial list either were or were not present based on the robot's responses. They observed the robot as it moved around on-screen.

The experimenter also explained the robot's capabilities. Participants were told that the robot could understand verbal instructions and requests, including moving to objects and turning. Participants knew that the robot could understand the referent keywords and movement instructions associated with them. The experimenter asked participants to speak to the robot clearly and at a normal pace. Participants were told that the robot would say as much as it understood and would try to get as close as possible to destinations.

Participants first did one training session with five practice tasks in order to learn how to interact with the robot. Practice involved moving the robot to specific landmarks in the environment using the identifier provided (e.g., using the keywords "blue lamp" in an instruction to move the robot to the next location). Participants continued until they completed all five practice tasks but were free to continue to move the robot around the environment until they were comfortable with the interface and setup.

The main experiment procedure is summarized in Figure 13. Following the training period, participants would complete an experiment session with the robot. The order of maps was randomized according to a Latin Square ${ }^{6}$ [38]. Participants would first instruct the robot through Session A, which contained five maps; each map had five trials. Each trial required the participant to check the existence of a referent by instructing the robot to navigate using the specified keyword. Since maps were composed of multiple trials, the participant needed to successfully move the robot to the next trial, specified by a referent subgoal, to proceed. After completing all trials in a map, the robot was moved to a new map. A session concluded after the fifth map was completed. Participants returned at a later time to complete Session B, which had maps that differed from Session A. They completed Sessions A and B three times; this full iteration is called a phase. We analyze a portion of the results from the study by phase.

\footnotetext{
${ }^{6}$ In Latin Square experiment design, stimuli are randomly sorted into a table in which rows represent trial ordering and columns represent repeated measures of the rows. The stimuli type appears once in each row and at most once in each column. Once a desired Latin Square is formed, the rows are first randomized, followed by columns. In this work, maps within a session were randomized according to a Latin Square.
} 

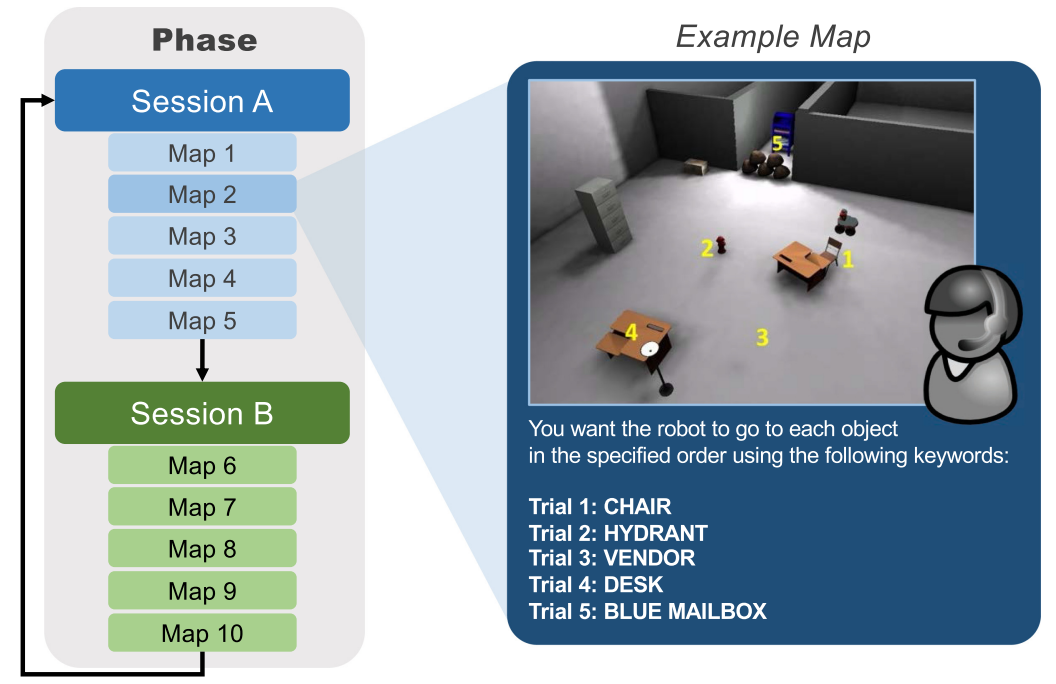

\begin{tabular}{|c|}
\hline Example Dialogue \\
\hline $\begin{array}{l}\text { Human: "Go to the chair." } \\
\text { Robot: "My map shows } \\
\text { more than one chair. Did } \\
\text { you mean the chair on my } \\
\text { right or the chair on my } \\
\text { left?" } \\
\text { H: "Chair on your left." } \\
\text { R: "Moving now." } \\
\text { <movement to chair> } \\
\text { R: "Finished." } \\
\text { H: "Move to the hydrant." } \\
\text { R: "Moving now." } \\
\text { <movement to hydrant> } \\
\text { R: "Finished." } \\
\text { H: "Go to the vendor." } \\
\text { R: "My map doesn't show } \\
\text { anything like that around } \\
\text { me." } \\
\text { H: "Go to the desk." } \\
\text { R: "My map shows more } \\
\text { than one desk. Did you } \\
\text { mean..." }\end{array}$ \\
\hline
\end{tabular}

Fig. 13. Experiment procedure. Following a training period, participants provided instructions to a robot over the course of ten different environments. In total, participants repeated going through all maps three times.

Some trials were predefined as impossible or referentially ambiguous because participants and the robot had different maps. In these trials, the robot responded with a question that included some context of the situation and a recovery strategy. This was met with a verbal reply for which the participant was expected to expand on the previous instruction based on the robot's recovery strategy. This initiated a multi-turn dialogue that either ended with the robot arriving at the goal location or failing to do so. The robot could use multiple sources of knowledge to initiate a recovery strategy. As such, there was no "best" recovery strategy known in advance of the study. The purpose was to show that the instance-based recovery strategy selection algorithm could improve at selecting strategies as the robot logged more interactions.

8.1.2 Experiment Design. In this user study, we varied how much training data was used with the $k$-NN learning algorithm; this was done to determine reasonable quantities of data that could support recovery strategies in situated human-robot dialogue. We investigated two configurations for learning common in the dialogue systems literature: training with all data available (general model [101]) or training with a specific user's data in order to personalize the interaction (userspecific model, e.g., [23, 119]). Both model types have their potential benefits, with general models providing more examples for training an underlying machine-learning algorithm and user tailoring promising to have a more adaptive experience for the user. We studied the possible trade-off, as it is unknown which is more desirable in low-resource scenarios such as dialogue with a robot. Although general models may always be preferable when data is expensive to collect, adapting to the user could have a large impact on the quality of dialogue-people's preferences about the kinds of information that should be presented in recovery strategies have been shown to vary [73]. At the same time, in this domain it may be possible to learn user-specific models with a limited number of training examples.

Half of the participants interacted with the robot configured with the general model, while the other half interacted with the robot configured with the user-specific model. In the general-model condition, only the first participant in the first session interacted with a robot that was trained only with crowdsourced data. All of the following interactions were used by the general model. 
In the user-specific model condition, models started with the initial crowdsourced data but would update with observations from each user's own data.

8.1.3 Measures. The performance of the recovery strategy selection algorithm was assessed by collecting user interactions with the robot. The only independent variable in this study was the type of learning model, general or user-specific model. Dependent variables, described below, focus on dialogue efficiency. We measured variation between each phase through all 10 map types.

We hypothesize that all measures of dialogue efficiency will improve as the robot gathers more experience interacting with users in both conditions. Following the principle of least collaborative effort [27], dialogues will only be as long as necessary for task completion, and we expect that the robot will select recovery strategies that more quickly resolve references as more successful strategies are collected.

Failed Recoveries. For a study trial with referential ambiguity or an impossible task, we counted failed recoveries as any attempt by the robot to select a recovery strategy that did not result in successful completion of the task. Recovery strategies associated with learning were only those that included some feature about the surroundings to establish context (e.g., color to disambiguate a referent). The robot should learn to favor successful recoveries over time.

Dialogue Turns. We also tabulated the number of dialogue turns taken by the participant and robot to complete a trial. Dialogue turn count is a common measure of dialogue efficiency [118]. A change in conversational floor incremented the dialogue turn count. Turns were counted only within a trial and only after the participant's task-related instruction was correctly recognized by the robot. This allowed us to focus on situated dialogue recovery as opposed to speech recognition repairs.

Time on Task. We tabulate the time on task during trials, another common measure of dialogue efficiency [118].

8.1.4 Participation. We recruited six participants for this study that had no prior experience with TeamTalk. They each participated in six 30-minute periods in which they were required to complete one session, which had five maps (25 trials in total per session). In total, each participant completed three phases through all trials.

\subsection{Results}

We compared the two model types, general and user-specific, using measures that assessed dialogue efficiency between participants and the robot over time. While the measures were collected for every trial in the user study, we present results for only the trials that required use of the learning algorithm. These were all of the ambiguous trials ( 25 in total) and nine of the impossibleto-execute trials. This resulted in 612 spoken-dialogue trials across the six participants.

Measurements by phase are shown in Table 6. The user-specific models initially performed worse than the general model, as measured by higher counts for failed recoveries, dialogue turns, and time on task in Phase 1. In the end, however, the user-specific models outperformed the general model-by Phase 3, the user-specific models had fewer failed recoveries, fewer dialogue turns, and faster time on task.

We analyzed all measures by phase only (a repeated measure), by learning-model type only, and by an interaction of the two. We used a factorial mixed-effects analysis of variance model $^{7}$ for each case, with phase and learning-model type counted as fixed effects (phase, learning-model

\footnotetext{
${ }^{7}$ This approach computed standard least squares regression using reduced maximum likelihood (REML) [44].
} 
Table 6. Dialogue Efficiency Measures Recorded by Learning-Model Type and Data Collection Phase

\begin{tabular}{lcccc}
\hline Learning-Model Type & Phase & \# Failed Recoveries & \# Dialogue Turns & Time on Task (s) \\
\hline General & Phase 1 & 29 & 506 & 2643 \\
& Phase 2 & 29 & 480 & 2363 \\
& Phase 3 & 28 & 452 & 2324 \\
\hline User-Specific & Phase 1 & 38 & 522 & 3004 \\
& Phase 2 & 14 & 424 & $\mathbf{2 1 4 4}$ \\
& Phase 3 & $\mathbf{1 0}$ & $\mathbf{4 0 8}$ & 2228 \\
\hline
\end{tabular}

Bold represents best overall results in a phase.

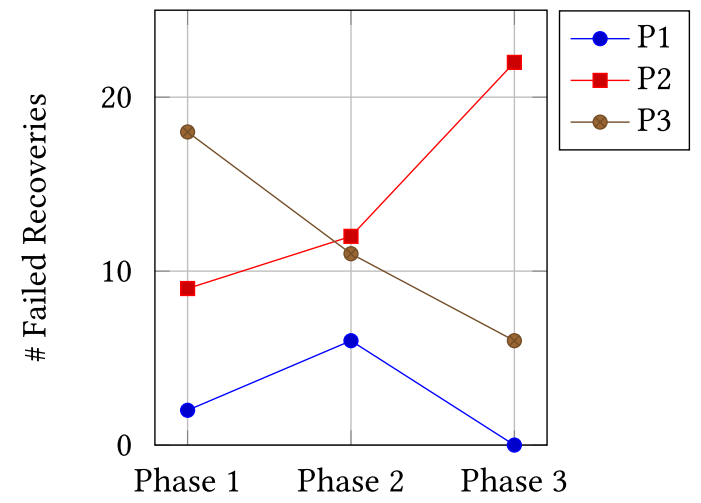

(a) General model results.

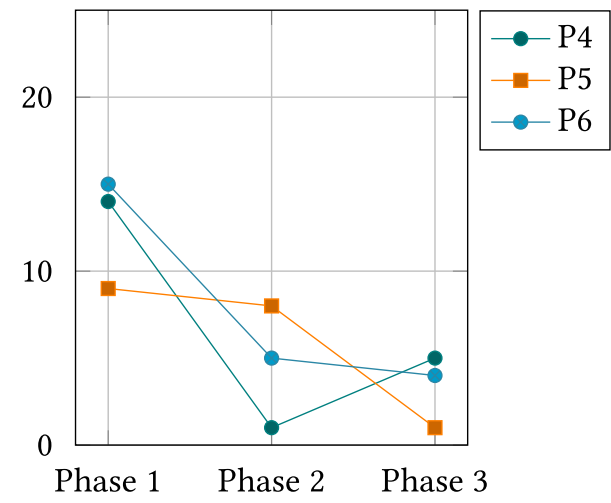

(b) User-specific model results.

Fig. 14. Participant results for the number of failed recoveries by phase.

type, and the interaction between them). Participant, nested by learning-model type, was included as a random effect in the model.

8.2.1 Failed Recoveries. All results for the number of failed recoveries by phase are presented in Table 6, with individual participant results in Figure 14. In total, the number of failed recoveries remained about the same for the general model, while those for the user-specific model decreased between phases. We predicted that the number of failed recoveries by the robot would decrease between phases for both models; the results support this hypothesis for the user-specific model only. With only the data from from the user-specific model condition, phase had a significant main effect $(\mathrm{F}[2,274]=5.8, p<0.005)$. The number of failed recoveries decreased most between the first phase and the second, and decreased again in the third phase. A Tukey HSD post-hoc test revealed that the Phase 1 measure was significantly different with Phase $2(p<0.05)$ and Phase $3(p<0.01)$. Significance results for user-specific models are summarized in Figure 15(a). No other significant main or interaction effects were found.

8.2.2 Dialogue Turns. Table 6 and Figure 16 summarize participant results for the number of dialogue turns by phase. Originally, we predicted that this value would decrease between phases. Results support this hypothesis for both the general and user-specific models. Phase had a significant main effect for results with both general and user-specific models $(\mathrm{F}[2,548]=5.1, p<0.01)$. The number of dialogue turns decreased significantly between Phases 1 and 3 of the study $(p<$ 0.01; Tukey HSD). Again analyzing only the user-specific data, there was a significant main effect for phase $(\mathrm{F}[2,82]=6.0, p<0.005)$. Phase 1 had more turns than Phase $2(p<0.05$; Tukey HSD $)$ 


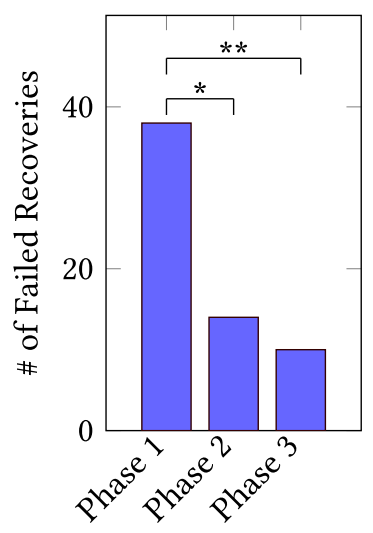

(a) Failed recoveries results.

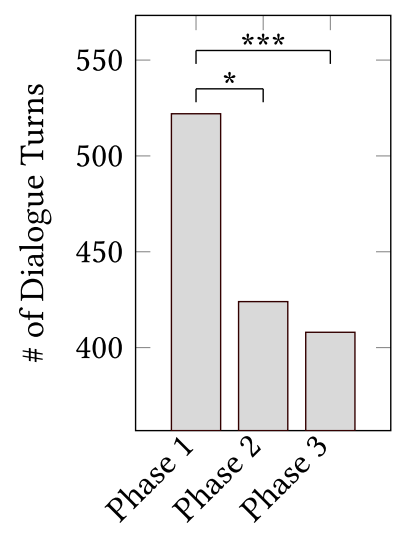

(b) Dialogue turn results.

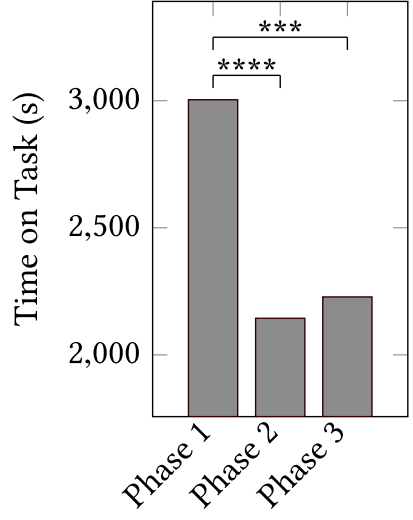

(c) Time on task results.

Fig. 15. For the user-specific learning-model type, dialogue efficiency significantly improved as the robot accumulated more experience with participants $\left({ }^{*} p<0.05 ;{ }^{* *} p<0.01 ;{ }^{* * *} p<0.005 ;{ }^{* * * *} p<0.001\right)$.

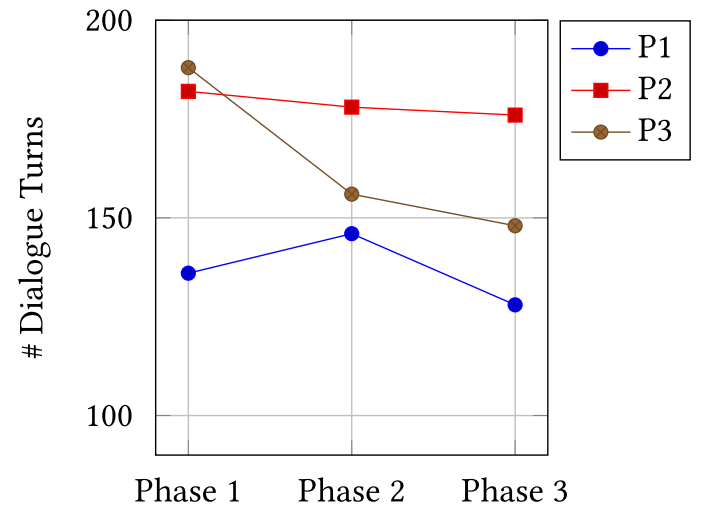

(a) General model results.

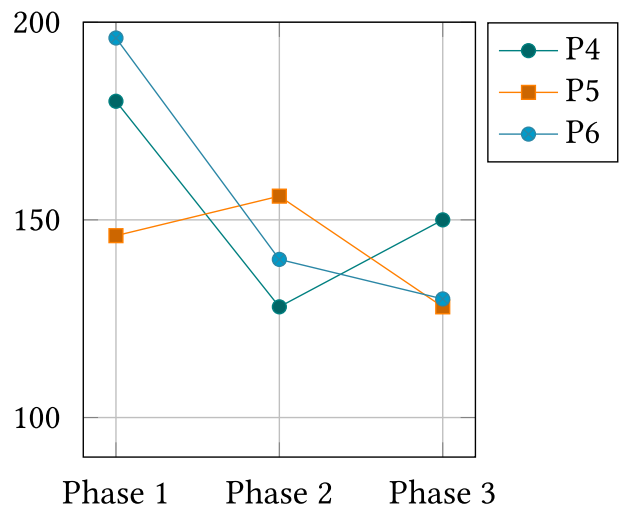

(b) User-specific model results.

Fig. 16. Participant results for the number of dialogue turns by phase.

and Phase 3 ( $p<0.005$; Tukey HSD). These significance results can be found in Figure 15(b). No other main or interaction effects were found.

8.2.3 Time on Task. Participant results for time on task by phase are shown in Table 6 and Figure 17. There was a decrease in time on task between most phases for both general and user-specific models. We predicted that time on task would decrease between phases for both learning-model types. Results support this hypothesis. Phase had a significant main effect on results with both general and user-specific models $(\mathrm{F}[2,548]=6.9, p<0.005)$. There was a significant decrease in time on task between Phase 1 and Phase $2(p<0.005$; Tukey HSD) and Phase 1 and Phase 3 ( $p<0.005$; Tukey HSD). No significant interactions were found. When analyzing only the user-specific data, phase had a significant main effect $(\mathrm{F}[2,274]=8.3, p<0.0005)$, again with Phase 1 having significantly longer time on task than Phase $2(p<0.001$; Tukey HSD) and Phase 3 $(p<0.005$; Tukey HSD). Significance results for user-specific models are presented in Figure 15(c). 


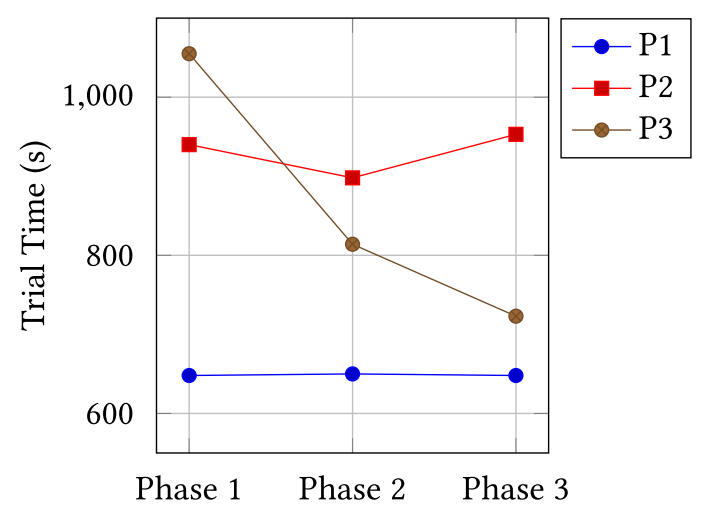

(a) General model results.

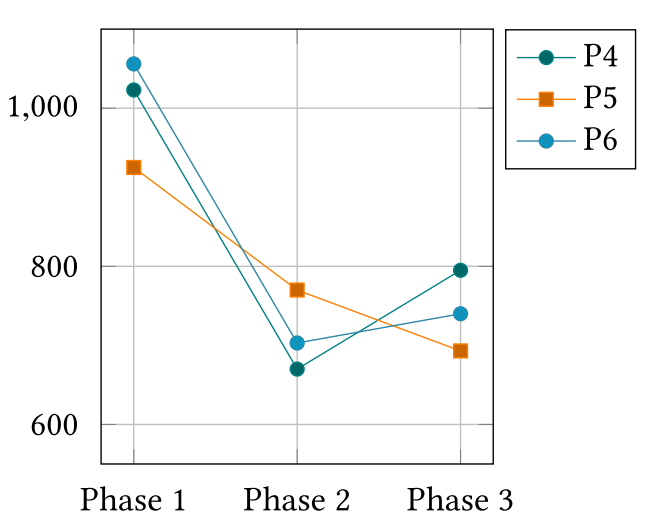

(b) User-specific model results.

Fig. 17. Participant results for time on task by phase.

\subsection{Discussion}

The results generally support our hypotheses, with the results for user-specific models better representing our predictions. Broadly speaking, the measures of dialogue efficiency improved as the robot accumulated experience with the task, with some exceptions.

User-specific models tended to result in stronger performance improvements over time compared with the general model that learned from all observations. We believe that this is because adapting to user preferences about environment content yields more successful dialogues than not doing so; preferences vary from user to user. Moreover, we find that users' preferences may differ from both the general model and the initial training data developed from crowdsourced recovery strategies, impacting the pace and progress of learning. We discuss the results for each measure below.

8.3.1 Failed Recoveries. A post-hoc evaluation shows that dialogues had a significant reduction in failed recoveries between the first and later phases for the user-specific condition. Note first that results on the failed recovery measure differ when comparing the first phase of the general model with those of the combined user-specific models. As seen in Table 6, there is a stark contrast in the number of failed recoveries for the general model compared with the user-specific ones; the user-specific ones have nine more failed recoveries (31\% more). Although there may be some user variance, the models collect observations differently. The general model accumulates observations of all participants in an online fashion; in the first phase, all users except for the very first interact with a robot that has experience with real-time recovery. In one sense, the user-specific models were handicapped by design: they all start with only the crowdsourced training data. In effect, the user-specific models adapt from the initial data for each user. The general model always has a larger training data size than the user-specific ones because it includes observations of all users; thus, the initial difference in failed recoveries is not a surprise.

The results also suggest that user-specific models are preferable when the interaction is longitudinal, such as with trained operators who are in long-term interactions with a particular robot. When the learning algorithm is collecting data about all users, it may negatively impact performance with people whose preferences deviate from the norm. For example, someone with a high spatial ability may prefer to use egocentric spatial terms such as "right" and "left" to differentiate possible referents; a similar phenomenon has been observed in human-human communication [99]. 
8.3.2 Dialogue Turns. Results also support our prediction that the number of dialogue turns would decrease between phases for both learning-model types. Phase had a significant main effect, with dialogues reducing in turn count over time. We note that there are no significant interactions, which suggests that neither learning-model type was optimizing better than the other for turn count. However, the user-specific models have the strongest performance improvements: there is a statistically significant reduction in dialogue turns from the first to second phases. The second and third phases are not significantly different, which suggests that the learning algorithm converged on an improved strategy set for users by the second phase.

8.3.3 Time on Task. Finally, results support our prediction that time on task would decrease between phases. We found that phase again had a significant main effect. Similar to dialogue turn count, we did not find any significant interactions between phase and learning model type, suggesting that neither learning model type is optimizing better than the other for time on task. We echo observations made in the failed recoveries discussion when considering the initial differences in performance between the two model types (see Table 6). As before, we attribute the differences to user variance and the differences in training data size between the general and user-specific models. Post-hoc evaluations support the existing evidence that the user-specific model is performing better between phases: the user-specific models have a statistically significant reduction in dialogue turns from the first to the second phase. Combined with values obtained for dialogue turn count, the results suggest that the learning algorithm is making improved choices over time.

8.3.4 Limitations. As with most repeated-measures experiments, there is the possibility of a learning effect since participants completed three iterations through all maps. Most of the dialogue efficiency measures improved as the robot engaged in more interactions with participants. However, we note several measures that were put into place to mitigate possible adverse learning effects. First, we conducted a series of practice trials using a map that was different from the study trials. This map had examples of both referentially ambiguous and impossible-to-execute instructions; thus, participants knew the general problem space. Participants would proceed to the main trials only after they felt comfortable with both the task and speaking with the system. Before issuing commands in a map, participants also saw an overview of the robot's last known surroundings; thus, they already had some familiarity with the environments. However, owing to task design, these differed from the robot's internal map in some ways. We also note that for each trial, participants knew what to instruct the robot about and knew the relevant terms that the robot understood for the objects in the scenes. The task completion rate was consistently high. To reduce ordering effects, we counterbalanced the order of maps for each participant according to a Latin Square. We expected minimal carry-over effects because participants had at least a day before they viewed the same trial.

An additional control condition in which the robot did not use the instance-based learning algorithm to select recovery strategies (e.g., selecting strategies at random or using heuristics) would have provided further evidence that there were no learning effects. The current results lack a nolearning condition that may have made it possible for dialogue efficiency measures to improve based entirely on the participant's ability to adapt to the robot.

\section{EVALUATING RECOVERY LEARNING WITH CROWDSOURCED DATA}

We evaluated the learning algorithm using various training data sizes with a held-out test set. This was done for two reasons: (1) to demonstrate that, despite a limited number of training examples, performance was improving as more recoveries were collected; and (2) to determine whether the feature space was appropriate for representing situations in the task. This is especially relevant given the high cost of recording situated dialogue. 
Upon completion of the longitudinal user study in the previous section, we collected 605 new training examples, 464 for successfully recovered ambiguous situations and 141 for successfully recovered impossible ones. We evaluated the two model types, general and user-specific, at two benchmarks: $50 \%$ and $100 \%$ of the final collected training data. We assessed the performance of the models to select recovery strategies for referential ambiguity and impossible-to-execute instructions separately. Given the results observed in the user study, we hypothesize that the model performance for both general and user-specific models will perform better with all of the training data than the $50 \%$ benchmark.

We used the crowdsourced test set withheld in Section 7.1.2 as our evaluation set; it reflects human judgments on which properties of the environment the robot should include in its clarifications (i.e., the learning task). The crowdsourced data was structured the same as the training data, with rows populating all features. For this evaluation, we measured the agreement rate of models with the test data at the $50 \%$ and $100 \%$ configurations of training data size. As such, we tabulated the number of times the models would select the class of environment properties (i.e., one of \{intrinsic (color), intrinsic (size), object proximity, egocentric spatial relation\}) that human judges included in the recovery strategies observed in the test set.

\subsection{Method}

Given the full set of crowdsourced recovery strategies that were collected in Section 7.1.2, we withheld a portion for testing (33\% of the original dataset, 131 instances; of which 108 were referentially ambiguous and 23 impossible tasks). Test set examples described a situation with a situated grounding problem and had a human's judgment on what type of strategy to take. An example test situation was: ROBOT IS POINTING FORWARD, BLUE BOX ON RIGHT, BROWN BOX ON LEFT, STIMULUS COMMAND Is "Move to the box." These data were represented the same way as the user study: a feature vector with all features that numerically represented the command and environment context for a situation, along with a human judge's strategy (i.e., class label) for that situation. For example, if a human judge entered, "Did you mean the box on the left or the right?" in the example above, the class label was annotated as an egocentric spatial relation. Since this was the only way to represent such a situation in the crowdsourced dataset, the same feature vector (representing the environment) was matched to up to 14 other human judges for that situation. All of the human judges viewed the same set of situations and heard the same synthesized commands.

We evaluated four models and two baselines. The general model used all training data at the $50 \%$ and $100 \%$ benchmarks, while the three user-specific models (one per participant) used only that participant's data at the $50 \%$ and $100 \%$ benchmarks. A random baseline would select recovery strategies at random, while a majority baseline would always select the most commonly used recovery strategy in the training data.

In the user study, the learning algorithm combined crowdsourced instances with new ones that it accumulated as the robot interacted with participants. However, since the test data does not contain spoken language features (commands were predefined), the learning algorithm may simply favor the crowdsourced data because it kept several language-specific features, such as speech recognition confidence, constant. To mitigate the favoring of the crowdsourced data and to assess the learning algorithm's ability to accumulate novel situations, we removed the initial crowdsourced data from the training set-it was meant only to bootstrap the initial data collection. The remaining training data was collected exclusively from spoken-dialogue interactions in the user study.

\subsection{Results}

We compared the general and user-specific models at training data intervals of $50 \%$ and $100 \%$ to evaluate whether performance would improve with more observations. In addition, we compared 
Table 7. Agreement Results on the Held-out Test Set Evaluation for General and User-Specific Models

\begin{tabular}{lcccccc}
\hline Learning Model & $\begin{array}{c}50 \% \text { Training } \\
\text { (all) }\end{array}$ & $\begin{array}{c}\text { 100\% Training } \\
\text { (all) }\end{array}$ & $\begin{array}{c}50 \% \text { Training } \\
\text { (ambiguous) }\end{array}$ & $\begin{array}{c}\text { 100\% Training } \\
\text { (ambiguous) }\end{array}$ & $\begin{array}{c}50 \% \text { Training } \\
\text { (impossible) }\end{array}$ & $\begin{array}{c}100 \% \text { Training } \\
\text { (impossible) }\end{array}$ \\
\hline Random & $26 \%$ & $26 \%$ & $26 \%$ & $26 \%$ & $26 \%$ & $26 \%$ \\
Majority & $44 \%$ & $47 \%$ & $44 \%$ & $44 \%$ & $48 \%$ & $61 \%$ \\
\hline General & $\mathbf{5 6 \%}$ & $\mathbf{6 6 \%}$ & $\mathbf{5 6 \%}$ & $\mathbf{6 6 \%}$ & $61 \%$ & $70 \%$ \\
User Model 1 & $40 \%$ & $51 \%$ & $38 \%$ & $48 \%$ & $48 \%$ & $70 \%$ \\
User Model 2 & $42 \%$ & $58 \%$ & $34 \%$ & $55 \%$ & $\mathbf{7 8 \%}$ & $\mathbf{7 4 \%}$ \\
User Model 3 & $33 \%$ & $50 \%$ & $27 \%$ & $47 \%$ & $61 \%$ & $61 \%$ \\
\hline Total Situations & 131 & 131 & 108 & 108 & 23 & 23 \\
\hline
\end{tabular}

We also report model agreement with random and majority baselines. Bold represents best-performing results.

each of the model types with the baselines to identify the best-performing model. Results are summarized in Table 7. We made the gold standard assumption that the human judges' preferences for recovery strategies in the test set were the correct ones. We report the student's $t$-test of proportions to assess statistical significance.

9.2.1 Training Data Size. We predicted that both the general and user-specific models would yield better agreement rates with more training data. The results confirmed this hypothesis. A student's $t$-test compared the accuracies for all trained models at $50 \%$ and $100 \%$ training data intervals. There was a significant difference in accuracies between the $50 \%(\mathrm{M}=0.43, \mathrm{SD}=0.10)$ and $100 \%(\mathrm{M}=0.53, \mathrm{SD}=0.08)$ training data sizes; $\mathrm{t}(3)=2.2, p<0.05$. Overall, the models configured with $100 \%$ training data significantly outperformed those with $50 \%$ training data.

9.2.2 Individual Model Performance. We first compared the general model results with the random and majority class baselines. Student's $t$-tests confirmed a significant difference between the random baseline $(\mathrm{M}=0.26, \mathrm{SD}=0.00)$ and general model $(\mathrm{M}=0.61, \mathrm{SD}=0.07) ; \mathrm{t}(1)=7.0, p<0.05$, as well as between the majority baseline $(\mathrm{M}=0.46, \mathrm{SD}=0.02)$ and the general model; $\mathrm{t}(1)=3.0, p<$ 0.05 .

Next, we compared the user-specific model results with the random and majority class baselines. Student's $t$-tests also confirmed a significant difference between the random baseline and userspecific models $(\mathrm{M}=0.46, \mathrm{SD}=0.09) ; \mathrm{t}(1)=2.9, p<0.05$. However, no significant difference was found when comparing the user-specific models with the majority baseline. We observed that the user-specific models all underperformed with the majority baseline at $50 \%$ training data but improved with $100 \%$ training data. Additionally, User Model 2 and User Model 3 for impossible recovery did not improve with more training data. The reduction in performance for User Model 2 was due to one fewer test case being correct.

Finally, we compared the general model to the user-specific models. A student's $t$-test found that the general model significantly outperformed the user-specific models on the test data; $t(1)=2.2, p<0.05$. Overall, the general model was the best performer on the test data.

\subsection{Discussion}

The results support our hypothesis that more data results in better performance for both general and user-specific models. This suggests that as the models accumulated more training examples, they tended to behave closer to human judges' preferences. We also found the general model to significantly outperform the baselines and user-specific model type when evaluating all test data. This may validate the general preferences collected from the crowdsourced study as representative of a more general population, as more data meant that the models' results aligned better with the 
test set (i.e., gold standard labels). Exceptional cases were two user models in which performance either did not improve or became marginally worse.

Results validate the learning approach and feature set. With the features investigated in this work, the learning models better aligned with human judges' preferences when they used more training examples. We find the feature set to be feasible for numerically representing situations in the navigation domain.

9.3.1 Limitations. We assumed that human judges' preferences for recovery strategies were correct, as we considered their data to be the gold standard in our evaluation. This is not necessarily true because every trial permitted multiple valid responses for recovery using properties of the robot's surroundings. In this work, agreement served as an estimate of general preference for a segment of the general population.

\section{CONCLUSIONS AND FUTURE WORK}

We described an infrastructure and representation for detecting and recovering from dialogue with situated grounding problems, cases in which physical agents such as robots interpret instructions that are referentially ambiguous or impossible to accomplish in their surroundings. We defined the problem space in the context of a directed navigation task in which a human gives instructions and a robot follows them, asking for clarification when appropriate. We then described core capabilities that robots must have in order to handle situated grounding problems. These capabilities were implemented in TeamTalk, a conversational interface with robots. We presented a rich representation that combines spoken-language input with static and dynamic sources of environment context. The TSR was developed to reason over this information and detect miscommunication events in situated human-robot dialogue. We also introduced a set of recovery strategies, motivated by observations in human-human dialogue, that robots can use by considering properties in their surroundings that can be included in clarification requests. Finally, we presented a method to improve recovery strategy selection using nearest-neighbor learning with data originally collected from crowdsourced workers and supplemented by real-time interactions with users. Our results showed that a robot could learn how to select human-inspired recovery strategies using a numerical representation of situations. We found that while population-level learning yielded the highest agreement with human judges, user-tailored learning led to more efficient successful dialogues in a longitudinal user study with six participants. We believe that these findings are relevant to the design of human-robot interfaces that use experience to improve performance.

There are many opportunities for future work in this area. While the contributions in this work enable recovery from situated grounding problems, the robot did not acquire new knowledge about the environment or actions beyond whether or not a selected strategy was successful. A desirable extension would be to update the robot's understanding of its physical surroundings in natural language. We made the simplifying assumption that the robot had knowledge about its surroundings encoded a priori. Future work can consider incorporating sources of uncertainty to help with selecting recovery strategies. This can include exploring evidence such as temporal information and previous exchanges. The impact of the nearest-neighbor learning algorithm could be made stronger by experimenting with an additional condition in a future study in which no learning takes place, instead basing the robot's decisions on heuristics or selecting them at random. Additionally, there remain other measures for route instruction interpretation that can be used to assess the positive impact of recovery strategies; these include effectiveness metrics common in the robotics literature, such as time to the goal, percentage of the route achieved, and adherence to a specified route. As the natural-language understanding in this work was designed based on existing dialogue data, there remain opportunities to automatically learn such interpretations from 
annotated training examples. Finally, there are many tasks that robots can accomplish with people that will require recovery from miscommunication, including manipulating objects, recurring tasks such as patrol, and interpreting the personal factors of dialogue partners. All of these tasks will require robots to interpret, reason, and communicate about the physical world with others.

\section{ACKNOWLEDGMENTS}

The authors thank Judith Klavans, Alan W. Black, Justine Cassell, Stephen LaRocca, Matthias Scheutz, and the anonymous reviewers for their helpful comments, and Clare Voss for her support of this work. The authors also thank Thomas K. Harris, Aasish Pappu, and Benjamin Frisch for their previous contributions to developing the TeamTalk dialogue platform.

\section{REFERENCES}

[1] Anne H. Anderson, Miles Bader, Ellen Gurman Bard, Elizabeth Boyle, Gwyneth Doherty, Simon Garrod, Stephen Isard, Jacqueline Kowtko, Jan McAllister, Jim Miller, Catherine Sotillo, Henry S. Thompson, and Regina Weinert. 1991. The HCRC map task corpus. Language and Speech 34, 4 (1991), 351-366.

[2] Giulio Antoniol, Bruno Caprile, Alessandro Cimatti, and Roberto Fiutem. 1994. Experiencing real-life interactions with the experimental platform of MAIA. In Proceedings of the 1st European Workshop on Human Comfort and Security.

[3] Yoav Artzi and Luke Zettlemoyer. 2013. Weakly supervised learning of semantic parsers for mapping instructions to actions. Transactions of the Association for Computational Linguistics 1 (2013), 49-62.

[4] Hideki Asoh, Yoichi Motomura, Futoshi Asano, Isao Hara, Satoru Hayamizu, Katsunobu Itou, Takio Kurita, Toshihiro Matsui, Nikos Vlassis, Roland Bunschoten, and Ben Kröse. 2001. Jijo-2: An office robot that communicates and learns. IEEE Intelligent Systems 16, 5 (2001), 46-55.

[5] Stephen Balakirsky, Chris Scrapper, and Elena Messina. 2005. Mobility open architecture simulation and tools environment. In Proceedings of the Knowledge Intensive Multi-Agent Systems Conference (KIMAS'05).

[6] Dhananjay Bansal and Mosur K. Ravishankar. 1998. New features for confidence annotation. In Proceedings of the 5th International Conference on Spoken Language Processing (ICSLP'98).

[7] Emanuele Bastianelli, Giuseppe Castellucci, Danilo Croce, Roberto Basili, and Daniele Nardi. 2017. Structured learning for spoken language understanding in human-robot interaction. The International fournal of Robotics Research 36, 5-7 (2017), 660-683.

[8] Samuel Bayer, Christine Doran, and Bryan George. 2001. Exploring speech-enabled dialogue with the Galaxy communicator infrastructure. In Proceedings of the 1st International Conference on Human Language Technology Research (HLT'01).

[9] Yonatan Bisk, Deniz Yuret, and Daniel Marcu. 2016. Natural language communication with robots. In Proceedings of NAACL-HLT'16.

[10] Dan Bohus. 2007. Error Awareness and Recovery in Conversational Spoken Language Interfaces. Ph.D. Dissertation. Carnegie Mellon University, Pittsburgh, PA.

[11] Dan Bohus and Eric Horvitz. 2010. On the challenges and opportunities of physically situated dialog. In Proceedings of the AAAI Fall Symposium on Dialog with Robots.

[12] Dan Bohus, Antoine Raux, Thomas K. Harris, Maxine Eskenazi, and Alexander I. Rudnicky. 2007. Olympus: An open-source framework for conversational spoken language interface research. In Proceedings of the NAACL-HLT Workshop on Bridging the Gap: Academic and Industrial Research in Dialog Technologies.

[13] Dan Bohus and Alexander I. Rudnicky. 2002. Integrating Multiple Knowledge Sources for Utterance-Level Confidence Annotation in the CMU Communicator Spoken Dialog System. Technical Report. Carnegie Mellon University, Pittsburgh, PA.

[14] Dan Bohus and Alexander I. Rudnicky. 2009. The RavenClaw dialog management framework: Architecture and systems. Computer Speech and Language 23, 3 (2009), 332-361.

[15] Dan Bohus, Chit W. Saw, and Eric Horvitz. 2014. Directions robot: In-the-wild experiences and lessons learned. In Proceedings of the 13th International Conference on Autonomous Agents and Multiagent Systems (AAMAS'14).

[16] Abdeslam Boularias, Felix Duvallet, Jean Oh, and Anthony Stentz. 2016. Learning qualitative spatial relations for robotic navigation. In Proceedings of the 25th International foint Conference on Artificial Intelligence (IfCAI'16).

[17] Gies Bouwman, Janienke Sturm, and Louis Boves. 1999. Incorporating confidence measures in the Dutch train timetable information system developed in the ARISE project. In Proceedings of the IEEE International Conference on Acoustics, Speech, and Signal Processing (ICASSP'99). 
[18] Jack E. Bresenham. 1965. Algorithm for computer control of a digital plotter. IBM Systems fournal 4, 1 (1965), 25-30.

[19] Alexander Broad, Jacob Arkin, Nathan Ratliff, Thomas Howard, and Brenna Argall. 2017. Real-time natural language corrections for assistive robotic manipulators. The International fournal of Robotics Research 36, 5-7 (2017), 684-698.

[20] Guido Bugmann, Ewan Klein, Stanislao Lauria, and Theocharis Kyriacou. 2004. Corpus-based robotics: A route instruction example. In Proceedings of Intelligent Autonomous Systems.

[21] Cesar Cadena, Luca Carlone, Henry Carrillo, Yasir Latif, Davide Scaramuzza, José Neira, Ian Reid, and John J. Leonard. 2016. Past, present, and future of simultaneous localization and mapping: Towards the robust-perception age. IEEE Transactions on Robotics 32, 6 (2016), 1309-1332.

[22] Stefano Carpin, Mike Lewis, Jijun Wang, Stephen Balakirsky, and Chris Scrapper. 2007. USARSim: A robot simulator for research and education. In Proceedings of the IEEE International Conference on Robotics and Automation (ICRA'07).

[23] Iñigo Casanueva, Thomas Hain, Heidi Christensen, Ricard Marxer, and Phil Green. 2015. Knowledge transfer between speakers for personalised dialogue management. In Proceedings of the 16th Annual SIGdial Meeting on Discourse and Dialogue (SIGdial'15).

[24] Joyce Y. Chai, Rui Fang, Changsong Liu, and Lanbo She. 2016. Collaborative language grounding toward situated human-robot dialogue. AI Magazine 37, 4 (2016), 32-45.

[25] Lin Chase. 1997. Error-Responsive Feedback Mechanisms for Speech Recognizers. Ph.D. Dissertation. Carnegie Mellon University, Pittsburgh, PA.

[26] David L. Chen and Raymond J. Mooney. 2011. Learning to interpret natural language navigation instructions from observations. In Proceedings of the 25th AAAI Conference on Artificial Intelligence (AAAI'11).

[27] Herbert H. Clark. 1996. Using Language. Cambridge University Press, New York, NY.

[28] Herbert H. Clark and Susan E. Brennan. 1991. Grounding in communication. Perspectives on Socially Shared Cognition. 127-149.

[29] Stephen Cox and Richard Rose. 1996. Confidence measures for the SWITCHBOARD database. In Proceedings of the IEEE International Conference on Acoustics, Speech, and Signal Processing (ICASSP'96).

[30] Robin Deits, Stefanie Tellex, Pratiksha Thaker, Dimitar Simeonov, Thomas Kollar, and Nicholas Roy. 2013. Clarifying commands with information-theoretic human-robot dialog. Journal of Human-Robot Interaction 2, 2 (2013), 58-79.

[31] Felix Duvallet, Matthew R. Walter, Thomas Howard, Sachithra Hemachandra, Jean Oh, Seth Teller, Nicholas Roy, and Anthony Stentz. 2016. Inferring maps and behaviors from natural language instructions. In Proceedings of the International Symposium on Experimental Robotics (ISER'16).

[32] Juraj Dzifcak, Matthias Scheutz, Chitta Baral, and Paul Schermerhorn. 2009. What to do and how to do it: Translating natural language directives into temporal and dynamic logic representation for goal management and action execution. In Proceedings of the IEEE International Conference on Robotics and Automation (ICRA'09).

[33] Juan Fasola and Maja J. Matarić. 2013. Using semantic fields to model dynamic spatial relations in a robot architecture for natural language instruction of service robots. In Proceedings of the IEEE/RSf International Conference on Intelligent Robots and Systems (IROS'13).

[34] Terrence Fong, Charles Thorpe, and Charles Baur. 2003. Collaboration, dialogue, and human-robot interaction. In Robotics Research. 255-266.

[35] Mary Ellen Foster, Rachid Alami, Olli Gestranius, Oliver Lemon, Marketta Niemelä, Jean-Marc Odobez, and Amit Kumar Pandey. 2016. The MuMMER project: Engaging human-robot interaction in real-world public spaces. In Proceedings of the International Conference on Social Robotics (ICSR'16).

[36] Mary Ellen Foster, Manuel Giuliani, Amy Isard, Colin Matheson, Jon Oberlander, and Alois Knoll. 2009. Evaluating description and reference strategies in a cooperative human-robot dialogue system. In Proceedings of the 21st International foint Conference on Artificial Intelligence (IFCAI'09).

[37] Benjamin Fransen, Vlad Morariu, Eric Martinson, Samuel Blisard, Matthew Marge, Scott Thomas, Alan Schultz, and Dennis Perzanowski. 2007. Using vision, acoustics, and natural language for disambiguation. In Proceedings of the 2nd ACM/IEEE International Conference on Human-Robot Interaction (HRI'07).

[38] Lei Gao. 2005. Latin Squares in Experimental Design. Technical Report. Michigan State University, East Lansing, MI.

[39] Genevieve Gorrell, Ian Lewin, and Manny Rayner. 2002. Adding intelligent help to mixed-initiative spoken dialogue systems. In Proceedings of the 7th International Conference on Spoken Language Processing (ICSLP'02).

[40] Edward T. Hall. 1966. The Hidden Dimension. Doubleday \& Co., New York, NY.

[41] Eui-Hong Han, George Karypis, and Vipin Kumar. 2001. Text categorization using weight adjusted $k$-nearest neighbor classification. In Proceedings of the Pacific-Asia Conference on Knowledge Discovery and Data Mining (PAKDD'01): Advances in Knowledge Discovery and Data Mining.

[42] Stevan Harnad. 1990. The symbol grounding problem. Physica D: Nonlinear Phenomena 42 (1990), 335-346.

[43] Thomas K. Harris and Alexander I. Rudnicky. 2007. TeamTalk: A platform for multi-human-robot dialog research in coherent real and virtual spaces. In Proceedings of the 22nd National Conference on Artificial Intelligence (AAAI'07). 
[44] David A. Harville. 1977. Maximum likelihood approaches to variance component estimation and to related problems. 7. Amer. Statist. Assoc. 72, 358 (1977), 320-338.

[45] Sachithra Hemachandra, Felix Duvallet, Thomas M. Howard, Nicholas Roy, Anthony Stentz, and Matthew R. Walter. 2015. Learning models for following natural language directions in unknown environments. In Proceedings of the IEEE International Conference on Robotics and Automation (ICRA'15).

[46] Julian Hough and David Schlangen. 2016. Investigating fluidity for human-robot interaction with real-time, realworld grounding strategies. In Proceedings of the 17th Annual SIGdial Meeting on Discourse and Dialogue (SIGdial'16).

[47] David Huggins-Daines, Mohit Kumar, Arthur Chan, Alan W. Black, Mosur Ravishankar, and Alexander I. Rudnicky. 2006. PocketSphinx: A free, real-time continuous speech recognition system for hand-held devices. In Proceedings of the IEEE International Conference on Acoustics, Speech, and Signal Processing (ICASSP'06).

[48] Ross A. Knepper, Stefanie Tellex, Adrian Li, Nicholas Roy, and Daniela Rus. 2015. Recovering from failure by asking for help. Autonomous Robots 39, 3 (2015), 347-362.

[49] Holger Knublauch, Ray W. Fergerson, Natalya F. Noy, and Mark A. Musen. 2004. The Protégé OWL plugin: An open development environment for semantic web applications. In Proceedings of the International Semantic Web Conference (ISWC'04).

[50] Thomas Kollar, Stefanie Tellex, Deb Roy, and Nicholas Roy. 2010. Toward understanding natural language directions. In Proceedings of the 5th ACM/IEEE International Conference on Human-Robot Interaction (HRI'10).

[51] Theodora Koulouri and Stanislao Lauria. 2009. A corpus-based analysis of route instructions in human-robot interaction. In Proceedings of Towards Autonomous Robotic Systems (TAROS'09).

[52] Emiel Krahmer, Marc Swerts, Mariet Theune, and Mieke Weegels. 2001. Error detection in spoken human-machine interaction. International fournal of Speech Technology 4 (2001), 19-30.

[53] Jayant Krishnamurthy and Thomas Kollar. 2013. Jointly learning to parse and perceive: Connecting natural language to the physical world. Transactions of the Association for Computational Linguistics 1 (2013), 193-206.

[54] Geert-Jan M. Kruijff, Pierre Lison, Trevor Benjamin, Henrik Jacobsson, and Nick A. Hawes. 2007. Incremental, multilevel processing for comprehending situated dialogue in human-robot interaction. In Proceedings of the Symposium on Language and Robots.

[55] Geert-Jan M. Kruijff, Pierre Lison, Trevor Benjamin, Henrik Jacobsson, Hendrik Zender, Ivana Kruijff-Korbayová, and Nick Hawes. 2010. Situated dialogue processing for human-robot interaction. Cognitive Systems (2010), 311-364.

[56] Geert-Jan M. Kruijff, Hendrik Zender, Patric Jensfelt, and Henrik I. Christensen. 2007. Situated dialogue and spatial organization: What, where... and why? International fournal of Advanced Robotic Systems 4, 1 (2007), 125-138.

[57] Stanislao Lauria, Guido Bugmann, Theocharis Kyriacou, Johan Bos, and Ewan Klein. 2002. Converting natural language route instructions into robot executable procedures. In Proceedings of the 11th IEEE International Workshop on Robot and Human Interactive Communication (RO-MAN'02).

[58] Séverin Lemaignan, Raquel Ros, Lorenz Mösenlechner, Rachid Alami, and Michael Beetz. 2010. ORO, a knowledge management platform for cognitive architectures in robotics. In Proceedings of the IEEE/RSf International Conference on Intelligent Robots and Systems (IROS 2'0).

[59] Séverin Lemaignan, Raquel Ros, E. Akin Sisbot, Rachid Alami, and Michael Beetz. 2012. Grounding the interaction: Anchoring situated discourse in everyday human-robot interaction. International fournal of Social Robotics 4, 2 (2012), 181-199.

[60] Oliver Lemon, Anne Bracy, Alexander Gruenstein, and Stanley Peters. 2001. A multi-modal dialogue system for human-robot conversation. In Proceedings of the 2nd North American Chapter of the Association for Computational Linguistics Conference (NAACL'01).

[61] Patrick Lester. 2005. A* Pathfinding for Beginners. https:/www.gamedev.net/articles/programming/artificialintelligence/a-pathfinding-for-beginners-r2003/.

[62] Michael Levit and Deb Roy. 2007. Interpretation of spatial language in a map navigation task. IEEE Transactions on Systems, Man, and Cybernetics, Part B (Cybernetics) 37, 3 (2007), 667-679.

[63] Diane J. Litman, Julia B. Hirschberg, and Marc Swerts. 2000. Predicting automatic speech recognition performance using prosodic cues. In Proceedings of the 1st North American Chapter of the Association for Computational Linguistics Conference (NAACL'00).

[64] Lorenzo Lucignano, Francesco Cutugno, Silvia Rossi, and Alberto Finzi. 2013. A dialogue system for multimodal human-robot interaction. In Proceedings of the 15th ACM International Conference on Multimodal Interaction (ICMI'13).

[65] James MacGlashan, Monica Babeş-Vroman, Marie desJardins, Michael L. Littman, Smaranda Muresan, Shawn Squire, Stefanie Tellex, Dilip Arumugam, and Lei Yang. 2015. Grounding English commands to reward functions. In Proceedings of Robotics: Science and Systems (RSS'15).

[66] Matt MacMahon, Brian Stankiewicz, and Benjamin Kuipers. 2006. Walk the talk: Connecting language, knowledge, and action in route instructions. In Proceedings of the 21st National Conference on Artificial Intelligence (AAAI'06). 
[67] Matthew Marge. 2015. Miscommunication Detection and Recovery for Spoken Dialogue Systems in Physically Situated Contexts. Ph.D. Dissertation. Carnegie Mellon University, Pittsburgh, PA.

[68] Matthew Marge, Aasish Pappu, Benjamin Frisch, Thomas K. Harris, and Alexander I. Rudnicky. 2009. Exploring spoken dialog interaction in human-robot teams. In Proceedings of Robots, Games, and Research: Success Stories in USARSim IROS Workshop.

[69] Matthew Marge, Aaron Powers, Jonathan Brookshire, Trevor Jay, Odest C. Jenkins, and Christopher Geyer. 2011. Comparing heads-up, hands-free operation of ground robots to teleoperation. In Proceedings of Robotics: Science and Systems (RSS'11).

[70] Matthew Marge and Alexander I. Rudnicky. 2010. Comparing spoken language route instructions for robots across environment representations. In Proceedings of the 11th Annual Meeting of the Special Interest Group on Discourse and Dialogue (SIGdial'10).

[71] Matthew Marge and Alexander I. Rudnicky. 2011. The TeamTalk corpus: Route instructions in open spaces. In Proceedings of the RSS Workshop on Grounding Human-Robot Dialog for Spatial Tasks.

[72] Matthew Marge and Alexander I. Rudnicky. 2011. Towards overcoming miscommunication in situated dialogue by asking questions. In Proceedings of the AAAI Fall Symposium on Building Representations of Common Ground with Intelligent Agents.

[73] Matthew Marge and Alexander I. Rudnicky. 2015. Miscommunication recovery in physically situated dialogue. In Proceedings of the 16th Annual Meeting of the Special Interest Group on Discourse and Dialogue (SIGdial'15).

[74] Cynthia Matuszek, Dieter Fox, and Karl Koscher. 2010. Following directions using statistical machine translation. In Proceedings of the 5th ACM/IEEE International Conference on Human-Robot Interaction (HRI'10).

[75] Cynthia Matuszek, Evan Herbst, Luke Zettlemoyer, and Dieter Fox. 2013. Learning to parse natural language commands to a robot control system. In Proceedings of the International Symposium on Experimental Robotics (ISER'13).

[76] Nikolaos Mavridis. 2015. A review of verbal and non-verbal human-robot interactive communication. Robotics and Autonomous Systems 63 (2015), 22-35.

[77] Nikolaos Mavridis and Deb Roy. 2006. Grounded situation models for robots: Where words and percepts meet. In Proceedings of the IEEE/RSF International Conference on Intelligent Robots and Systems (IROS'06).

[78] Raveesh Meena, José Lopes, Gabriel Skantze, and Joakim Gustafson. 2015. Automatic detection of miscommunication in spoken dialogue systems. In Proceedings of the 16th Annual Meeting of the Special Interest Group on Discourse and Dialogue (SIGdial'15).

[79] Raveesh Meena, Gabriel Skantze, and Joakim Gustafson. 2012. A data-driven approach to understanding spoken route directions in human-robot dialogue. In Proceedings of the 13th Annual Conference of the International Speech Communication Association (Interspeech'12).

[80] Raveesh Meena, Gabriel Skantze, and Joakim Gustafson. 2014. Data-driven models for timing feedback responses in a map task dialogue system. Computer Speech \& Language 28, 4 (2014), 903-922.

[81] Hongyuan Mei, Mohit Bansal, and Matthew R. Walter. 2016. Listen, attend, and walk: Neural mapping of navigational instructions to action sequences. In Proceedings of the 30th AAAI Conference on Artificial Intelligence (AAAI'16).

[82] Tom M. Mitchell. 1997. Machine Learning. McGraw Hill, New York, NY.

[83] Marius Muja and David G. Lowe. 2014. Scalable nearest neighbor algorithms for high dimensional data. IEEE Transactions on Pattern Analysis and Machine Intelligence 36, 11 (2014), 2227-2240.

[84] Aishwarya Padmakumar, Jesse Thomason, and Raymond J. Mooney. 2017. Integrated learning of dialog strategies and semantic parsing. In Proceedings of the 15th Conference of the European Chapter of the Association for Computational Linguistics (EACL'17).

[85] Tim Paek. 2003. Toward a taxonomy of communication errors. In Proceedings of the ISCA Workshop on Error Handling in Spoken Dialogue Systems.

[86] Ioannis Papaioannou and Oliver Lemon. 2017. Combining chat and task-based multimodal dialogue for more engaging HRI: A scalable method using reinforcement learning. In Proceedings of the Companion of the 2017 ACM/IEEE International Conference on Human-Robot Interaction (HRI LBR'17).

[87] Aasish Pappu and Alexander I. Rudnicky. 2012. The structure and generality of spoken route instructions. In Proceedings of the 13th Annual Meeting of the Special Interest Group on Discourse and Dialogue (SIGdial'12).

[88] Vittorio Perera and Manuela Veloso. 2015. Handling complex commands as service robot task requests. In Proceedings of the 24th International foint Conference on Artificial Intelligence (IFCAI'15).

[89] Dennis Perzanowski, Derek Brock, William Adams, Magdalena Bugajska, Alan C. Schultz, J. Gregory Trafton, Samuel Blisard, and Marjorie Skubic. 2003. Finding the FOO: A pilot study for a multimodal interface. In Proceedings of the IEEE International Conference on Systems, Man and Cybernetics (SMC'03).

[90] Vasumathi Raman, Constantine Lignos, Cameron Finucane, Kenton C. T. Lee, Mitch Marcus, and Hadas KressGazit. 2013. Sorry Dave, I'm afraid I can't do that: Explaining unachievable robot tasks using natural language. In Proceedings of Robotics: Science and Systems (RSS'13). 
[91] Antoine Raux and Maxine Eskenazi. 2004. Non-native users in the Let's Go!! Spoken dialogue system: Dealing with linguistic mismatch. In Proceedings of the Human Language Technology Conference of the North American Chapter of the Association for Computational Linguistics (HLT-NAACL'04).

[92] Antoine Raux and Maxine Eskenazi. 2008. Optimizing endpointing thresholds using dialogue features in a spoken dialogue system. In Proceedings of the 9th SIGdial Workshop on Discourse and Dialogue (SIGdial'08).

[93] Shaoqing Ren, Kaiming He, Ross Girshick, and Jian Sun. 2015. Faster R-CNN: Towards real-time object detection with region proposal networks. In Proceedings of the Neural Information Processing Systems Conference (NIPS'15).

[94] Stephanie Rosenthal, Joydeep Biswas, and Manuela Veloso. 2010. An effective personal mobile robot agent through symbiotic human-robot interaction. In Proceedings of the 9th International Conference on Autonomous Agents and Multiagent Systems (AAMAS'10).

[95] Alexander I. Rudnicky, Aasish Pappu, Peng Li, and Matthew Marge. 2010. Instruction taking in the TeamTalk system. In Proceedings of the AAAI Fall Symposium on Dialog with Robots.

[96] Rubén San-Segundo, Bryan Pellom, Wayne Ward, and José M. Pardo. 2000. Confidence measures for dialogue management in the CU communicator system. In Proceedings of the IEEE International Conference on Acoustics, Speech, and Signal Processing (ICASSP'00).

[97] Matthias Scheutz, Rehj Cantrell, and Paul Schermerhorn. 2011. Toward humanlike task-based dialogue processing for human robot interaction. AI Magazine 32, 4 (2011), 77-84

[98] David Schlangen. 2004. Causes and strategies for requesting clarification in dialogue. In Proceedings of the 5th SIGdial Workshop on Discourse and Dialogue (SIGdial'04).

[99] Michael F. Schober. 2009. Spatial dialogue between partners with mismatched abilities. Spatial Language and Dialogue. 23-39.

[100] Stephanie Schuldes, Michael Roth, Anette Frank, and Michael Strube. 2009. Creating an annotated corpus for generating walking directions. In Proceedings of the ACL-IFCNLP Workshop on Language Generation and Summarisation.

[101] Iulian Vlad Serban, Ryan Lowe, Peter Henderson, Laurent Charlin, and Joelle Pineau. 2018. A survey of available corpora for building data-driven dialogue systems: The journal version. Dialogue and Discourse 9, 1 (2018).

[102] Hui Shi and Bernd Krieg-Brückner. 2008. Modelling route instructions for robust human-robot interaction on navigation tasks. International fournal of Software and Informatics 2, 1 (2008), 33-60.

[103] Hui Shi and Thora Tenbrink. 2009. Telling Rolland where to go: HRI dialogues on route navigation. Spatial Language and Dialogue (2009), 177-189.

[104] Nobuyuki Shimizu and Andrew Haas. 2009. Learning to follow navigational route instructions. In Proceedings of the 21st International foint Conference on Artificial Intelligence (IFCAI'09).

[105] Gabriel Skantze. 2005. Exploring human error recovery strategies: Implications for spoken dialogue systems. Speech Communication 45, 3 (2005), 325-341.

[106] Gabriel Skantze. 2007. Error Handling in Spoken Dialogue Systems: Managing Uncertainty, Grounding and Miscommunication. Ph.D. Dissertation. KTH Royal Institute of Technology, Stockholm, Sweden.

[107] Gabriel Skantze. 2007. Making grounding decisions: Data-driven estimation of dialogue costs and confidence thresholds. In Proceedings of the 8th SIGdial Workshop on Discourse and Dialogue (SIGdial'07).

[108] Marjorie Skubic, Dennis Perzanowski, Samuel Blisard, Alan Schultz, William Adams, Magda Bugajska, and Derek Brock. 2004. Spatial language for human-robot dialogs. IEEE Transactions on Systems, Man, and Cybernetics, Part C: Applications and Reviews 34, 2 (2004), 154-167.

[109] Dimitris Spiliotopoulos, Ion Androutsopoulos, and Constantine D. Spyropoulos. 2001. Human-robot interaction based on spoken natural language dialogue. In Proceedings of the European Workshop on Service and Humanoid Robots.

[110] Laura Stoia, Darla Magdalene Shockley, Donna K. Byron, and Eric Fosler-Lussier. 2008. SCARE: A situated corpus with annotated referring expressions. In Proceedings of the Language Resources and Evaluation Conference (LREC'08).

[111] Stefanie Tellex, Thomas Kollar, Steven Dickerson, Matthew R. Walter, Ashis Gopal Banerjee, Seth Teller, and Nicholas Roy. 2011. Understanding natural language commands for robotic navigation and mobile manipulation. In Proceedings of the 25th AAAI Conference on Artificial Intelligence (AAAI'11).

[112] Thora Tenbrink, Robert J. Ross, Kavita E. Thomas, Nina Dethlefs, and Elena Andonova. 2010. Route instructions in map-based human-human and human-computer dialogue: A comparative analysis. fournal of Visual Languages \& Computing 21, 5 (2010), 292-309.

[113] Moritz Tenorth and Michael Beetz. 2013. KnowRob: A knowledge processing infrastructure for cognition-enabled robots. The International fournal of Robotics Research 32, 5 (2013), 566-590.

[114] Jesse Thomason, Shiqi Zhang, Raymond Mooney, and Peter Stone. 2015. Learning to interpret natural language commands through human-robot dialog. In Proceedings of the 24th International foint Conference on Artificial Intelligence (IFCAI'15). 
[115] Andrea Vanzo, Danilo Croce, Emanuele Bastianelli, Guglielmo Gemignani, Roberto Basili, and Daniele Nardi. 2017. Dialogue with robots to support symbiotic autonomy. In Dialogues with Social Robots. 331-342.

[116] Leslie Versweyveld. 1998. Voice-controlled surgical robot ready to assist in minimally invasive heart surgery. Virtual Medical Worlds Monthly.

[117] Adam Vogel and Dan Jurafsky. 2010. Learning to follow navigational directions. In Proceedings of the 48th Annual Meeting of the Association for Computational Linguistics (ACL'10).

[118] Marilyn A. Walker, Diane J. Litman, Candace A. Kamm, and Alicia Abella. 1998. Evaluating spoken dialogue agents with PARADISE: Two case studies. Computer Speech \& Language 12, 4 (1998), 317-347.

[119] Marilyn A. Walker, Stephen J. Whittaker, Amanda Stent, Preetam Maloor, Johanna Moore, Michael Johnston, and Gunaranjan Vasireddy. 2004. Generation and evaluation of user tailored responses in multimodal dialogue. Cognitive Science 28 (2004), 811-840.

[120] Marilyn A. Walker, Jerry Wright, and Irene Langkilde. 2000. Using natural language processing and discourse features to identify understanding errors in a spoken dialogue system. In Proceedings of the 17th International Conference on Machine Learning (ICML'00).

[121] Tom Williams and Matthias Scheutz. 2017. Resolution of referential ambiguity in human-robot dialogue using Dempster-Shafer theoretic pragmatics. In Proceedings of Robotics: Science and Systems (RSS'17).

[122] Tom Williams, Fereshta Yazdani, Prasanth Suresh, Matthias Scheutz, and Michael Beetz. 2018. Dempster-Shafer theoretic resolution of referential ambiguity. Autonomous Robots (2018), 1-26.

[123] Teresa Zollo. 1999. A study of human dialogue strategies in the presence of speech recognition errors. In Proceedings of the AAAI Fall Symposium on Psychological Models of Communication in Collaborative Systems.

Received April 2018; revised August 2018; accepted October 2018 